\title{
Desarguesian and Unitary complete partial ovoids
}

\author{
Valentina Pepe
}

Received: 24 October 2011 / Accepted: 21 April 2012 / Published online: 16 May 2012

(C) Springer Science+Business Media, LLC 2012

\begin{abstract}
The Desarguesian and Unitary ovoids of $\mathcal{Q}^{+}(7, q)$ are contained in a subvariety of the Grassmannian. We generalize this result, obtaining two families of complete partial ovoids of polar spaces: the first, with stabilizer PGL $\left(2, q^{t}\right)$ and including the family constructed by A. Cossidente and O.H. King for even $q$, and the second, with stabilizers contained in $\mathrm{PGU}(r, q)$.
\end{abstract}

Keywords Polar space · Quadric · Complete partial ovoid · Twisted tensor embedding · Grassmannian

\section{Introduction}

Ovoids are among the most widely investigated structures of polar spaces. In the case of $\mathcal{Q}^{+}(7, q)$, three infinite families are known: the Desarguesian [16], with $q$ even and stabilizer PGL $\left(2, q^{3}\right)$, the Unitary [16, 31], with $q \equiv 0,2 \bmod 3$ and stabilizer PGU $(3, q)$, and the Ree-Tits [36] with $q=3^{2 h+1}$ and stabilizer ${ }^{2} G_{2}(q)$. We are focused on two of the aforementioned families: the Desarguesian and the Unitary one. We want to recall a way to construct them that is alternative with respect to the original constructions. In [20], it has been pointed out for the first time that the Desarguesian ovoid of $\mathcal{Q}^{+}(7, q), q$ even, consists of the set of the points of the Grassmannian that are image of the Desarguesian plane spread of PG $(5, q)$; then, in [5], with a different terminology and a more group-theoretic approach, this result has been extended, getting complete partial ovoids of $\mathcal{Q}^{+}\left(2^{t}-1, q\right), t \geq 4$. In [21], it has been proven that a suitable hyperplane section of the Hermitian Veronesean, the image under the Grassmann map of the Desarguesian line spread of PG $(5, q)$, is the Unitary ovoid of $\mathcal{Q}^{+}(7, q), q \equiv 2 \bmod 3$, and the projection of such a set onto a

\footnotetext{
V. Pepe $(\bowtie)$

Department of Mathematics, Ghent University, Krijgslaan 281, Building S22, 9000 Gent, Belgium e-mail: valepepe@cage.ugent.be
} 
suitable space of codimension two is the Unitary ovoid of $\mathcal{Q}(6, q)$ (and hence also of $\left.\mathcal{Q}^{+}(7, q)\right)$, when $q=3^{h}$.

This paper is structured as follows. In Sect. 2, we recall some basic notions about polar spaces and review known results about their ovoids and partial ovoids. Then we describe the variety $\mathcal{V}_{r, t}$, which plays a fundamental role in our arguments: $\mathcal{V}_{r, t}$ is the image under the Grassmann map of the elements of a Desarguesian $(t-1)$-spread of $\mathrm{PG}(r t-1, q)$ and it is the geometric realization of the twisted tensor product group embedding. Section 3 is dedicated to the construction of Desarguesian complete partial ovoids of polar spaces of PG $\left(2^{t}-1, q\right)$, generalizing the constructions of [5] for $q$ even and the construction of [4] for $q$ odd and $t=3$. In Sect. 4, Unitary complete partial ovoids of quadrics are considered. Under the twisted tensor product representation, it is possible to embed points pairwise not collinear in $\mathcal{H}\left(r-1, q^{2}\right)$ into partial ovoids of a suitable quadric of PG $\left(r^{2}-1, q\right)$ contained in $\mathcal{V}_{r, 2}$, also called the Hermitian Veronesean. When $r=3$ and $q \equiv 0,2 \bmod 3$, this leads to the Unitary ovoid of $\mathcal{Q}^{+}(7, q)$. Our aim is to construct large complete partial ovoids of quadrics that are images under the twisted tensor embedding of ovoids of $\mathcal{H}\left(4, q^{2}\right)$.

\section{Preliminary results}

\subsection{Polar spaces}

Let $\mathrm{PG}(V, \mathbb{F})$ be the projective space defined by the lattice of subspaces of the vector space $V$ over the field $\mathbb{F}$; we assume that $\mathbb{F}$ is the finite field $\mathbb{F}_{q}, \operatorname{dim}_{\mathbb{F}_{q}} V=n$ and write $\operatorname{PG}(n-1, q):=\operatorname{PG}\left(V, \mathbb{F}_{q}\right), V(n, q):=V$. Let $\beta$ be a non-degenerate reflexive $\sigma$-sesquilinear form, $\sigma \in \operatorname{Aut}(\mathbb{F})$, defined on $V(n, \mathbb{F}) \times V(n, \mathbb{F})$, then $\perp: u \in V(n, \mathbb{F}) \mapsto\{v \in V \mid \beta(u, v)=0\} \subset V$ defines a polarity of $\mathrm{PG}(n-1, \mathbb{F})$. A subspace $W$ of $V$ is said to be totally isotropic if $\beta(u, v)=0$ for all $u$ and $v \in W$ and the lattice of totally isotropic subspaces is called a polar space (induced by $\beta$ or $\perp$ ); the subspaces of maximum dimension are called generators of the polar space. The form $\beta$ is said to be alternating, symmetric or hermitian, according to $\beta(u, u)=0$ for all $u \in V$ and $\sigma=i d, \beta(u, v)=\beta(v, u)$ for all $v, u \in V$ and $\sigma=i d, \beta(u, v)=\beta(v, u)^{\sigma}$ for all $v, u \in V$ and $\sigma^{2}=i d, \sigma \neq i d$ (and hence $\left.\mathbb{F}=\mathbb{F}_{q^{2}}\right)$, respectively. When $\beta$ is alternating, the associated polar space is said to be symplectic, $n$ must be even and the subgroup of $\operatorname{PGL}(n, q)$ preserving it is denoted by $\operatorname{PSp}(n, q)$; the dimension of a generator is $(n-2) / 2$. When $\beta$ is symmetric, then the polar space we obtain is said to be orthogonal, but we can regard this kind of polar space as also arising from a non-degenerate quadratic form $Q$ defined on $V$ : $\beta(u, v):=Q(u+v)-Q(u)-Q(v)$ is a non-degenerate symmetric bilinear form and the orthogonal polar space associated to it is given by the lattice of subspaces contained in the quadric $\mathcal{Q}$ defined by the equation $Q(v)=0$. We have to distinguish two cases: if $n$ is odd, then the dimension of a generator is $(n-1) / 2-1$, the quadric, denoted by $\mathcal{Q}(2 m, q), 2 m=n-1$, is said to be parabolic and the subgroup of $\operatorname{PGL}(n, q)$ fixing it is denoted by $\operatorname{PGO}(n, q)$; if $n$ is even, then there are, up to projective equivalence, two types of quadric, elliptic, denoted by $\mathcal{Q}^{-}(2 m+1, q)$, and hyperbolic, denoted by $\mathcal{Q}^{+}(2 m+1, q), 2 m+1=n-1$, according to whether 
they have complete subspaces of dimension $n / 2-1$ or $n / 2-2$, respectively, and the subgroups of PGL $(n, q)$ fixing them are, respectively, denoted by $\mathrm{PGO}^{-}(n, q)$ and $\mathrm{PGO}^{+}(n, q)$. Finally, when $\beta$ is hermitian, the associated polar space is said to be hermitian as well and a hermitian polar spaces can be seen as the lattice of subspaces contained in a non-singular Hermitian variety, denoted by $\mathcal{H}\left(n-1, q^{2}\right)$ and defined by the equation $\beta(v, v)=0$; in this case, the dimension of a generator is $\left\lfloor\frac{n-2}{2}\right\rfloor$ and the subgroup of $\operatorname{PGL}\left(n, q^{2}\right)$ preserving it is denoted by $\operatorname{PGU}(n, q)$.

We want to recall two important results about polar spaces. If $q$ is even and $n$ is odd, say $n=2 m+1$, then the orthogonal polar space of $\mathcal{Q}(2 m, q)$ is isomorphic to the symplectic polar space of PG $(2 m-1, q)$; moreover, for a fixed symplectic polarity $\perp$, there exists an elliptic quadric $\mathcal{Q}^{-}(2 m-1, q)$, and a hyperbolic quadric $\mathcal{Q}^{+}(2 m-1, q)$ inducing $\perp$ as well. In order to determine whether a certain quadric $\mathcal{Q}$ is elliptic or hyperbolic, we often use the following result. If $V=V_{1} \oplus V_{2} \oplus$ $\cdots \oplus V_{\frac{n}{2}}, V_{i} \cong V(2, q)$, and $\beta\left(v_{i}, v_{j}\right)=0$ for all $v_{i} \in V_{i}, v_{j} \in V_{j}, i \neq j$, then the associated polar space is said to be the orthogonal sum of the lines $\operatorname{PG}\left(V_{i}, q\right)$; the line is hyperbolic if it intersects $\mathcal{Q}$ in two points, the line is said to be non-isotropic if it is skew to $\mathcal{Q}$. Let $n^{\prime}$ be the number of non-isotropic lines occurring in the sum, then $\mathcal{Q}$ is hyperbolic if and only if $n^{\prime}$ is even. We refer to [9, 15, 29] for more about polar spaces.

An ovoid $\mathcal{O}$ of a polar space $\mathbb{P}$ is a set of points of $\mathbb{P}$ such that every generator meets $\mathcal{O}$ in exactly one point. If every generator meets $\mathcal{O}$ in at most one point, then $\mathcal{O}$ is called a partial ovoid. In both cases, for all $P_{1}$ and $P_{2} \in \mathcal{O}, P_{1} \neq P_{2}$, we have $P_{2} \notin P_{1}^{\perp}$, that is, the line $\left\langle P_{1}, P_{2}\right\rangle$ is not totally singular and $P_{1}$ and $P_{2}$ are said to be non-collinear in the polar space. The existence of ovoids is still an open problem for many polar spaces, while for several others, it is proven that they do not exist; in these latter cases, the existence and the size of complete partial ovoids is the most natural open problem: a partial ovoid is said to be complete if it is not properly contained in a larger one. For the size of an ovoid in a given polar space, we refer to [15, Appendix VI]. We present an overview of the results about ovoids of polar spaces. A symplectic polar space of PG $(2 n+1, q)$ has ovoids if and only if $n=1$ and $q$ is even (see [30, 32]). A Hermitian variety $\mathcal{H}\left(n-1, q^{2}\right)$ does not have ovoids if $n$ is odd [32], whereas if $n$ is even, there are constructions of ovoids only for $n=4$ and it is not known whether for $n>4$ and even the variety admits ovoids. Regarding $\mathcal{Q}^{-}(2 n+1, q)$, it is well known (see [32]) that it does not have ovoids for $n>1$. The quadric $\mathcal{Q}(4, q)$, has ovoids [24], but $\mathcal{Q}(2 n, q), n \geq 3$ and $q$ even, does not have ovoids (see [32]) and $\mathcal{Q}(2 n, q), n \geq 4$ and $q$ odd, does not have ovoids by [11]. Finally, $\mathcal{Q}(6, q)$ admits two families of ovoids in characteristic three [16,31], but it is still an open problem whether they exist or not in other odd characteristics. Regarding the hyperbolic quadric $\mathcal{Q}^{+}(2 n+1, q)$, the existence for ovoids is proven in all the characteristics only for $n \leq 2$; for $n \geq 4$, by [1], they do not exist if $q=p^{h}, p$ is a prime and $p^{n}>\left(\begin{array}{c}2 n+p \\ 2 n+1\end{array}\right)$, but it is not known whether they exist or not in the remaining cases. We are especially interested in $\mathcal{Q}^{+}(7, q)$. There are sporadic constructions for $q \geq 5$ a prime in [2, 22] and for $q=8$ in [10]. The infinite known families are: the Desarguesian [16], with $q$ even and stabilizer PGL $\left(2, q^{3}\right)$, the Unitary [16, 31], with $q \equiv 0,2 \bmod 3$ and stabilizer PGU $(3, q)$ and the Ree-Tits [36] with $q=3^{2 h+1}$ and stabilizer ${ }^{2} G_{2}(q)$. For $q$ not a prime and $q \equiv 1 \bmod 6$ is not known whether they exist or not. 


\subsection{The twisted tensor embedding}

A subset of PG $(n-1, q)$ isomorphic to $\mathrm{PG}\left(n-1, q^{\prime}\right), q^{\prime} \mid q$, is a subgeometry of PG $(n-1, q)$ and when $q=q^{\prime 2}$, then it is a Baer subgeometry. A subgeometry $\mathrm{PG}\left(n-1, q^{\prime}\right)$ can also be regarded as the set of fixed points with respect to a $\mathbb{F}_{q^{\prime}}$ linear collineation $\rho$ of PG $(n-1, q)$ of order $h=\log _{q^{\prime}} q$. In general, any subspace $\Pi$ of PG $(n-1, q)$ intersects PG $\left(n-1, q^{\prime}\right)$ in the subspace $\Pi \cap \Pi^{\rho} \cap \cdots \cap \Pi^{\rho^{h-1}}$, hence when $\Pi$ is set-wise fixed by $\rho$, then it meets PG $\left(n-1, q^{\prime}\right)$ in a space with the same dimension as $\Pi$.

Take PG $\left(r_{1}-1, q\right), \mathrm{PG}\left(r_{2}-1, q\right), \ldots, \mathrm{PG}\left(r_{t}-1, q\right)$ to be $t$ distinct projective spaces; the Segre embedding

$$
\begin{gathered}
\sigma: \quad \mathrm{PG}\left(r_{1}-1, q\right) \times \mathrm{PG}\left(r_{2}-1, q\right) \times \cdots \times \mathrm{PG}\left(r_{t}-1, q\right) \\
\quad \longrightarrow \mathrm{PG}\left(r_{1} r_{2} \cdots r_{t}-1, q\right)
\end{gathered}
$$

is the map such that $\sigma\left(\mathbf{x}^{1}, \ldots, \mathbf{x}^{t}\right)$ is the vector of all the possible products $x_{j_{1}}^{(1)} x_{j_{2}}^{(2)} \cdots x_{j_{t}}^{(t)}$, as $\mathbf{x}^{i}=\left(x_{0}^{(i)}, x_{1}^{(i)}, \ldots, x_{r_{i}-1}^{(i)}\right)$ varies in PG $\left(r_{i}-1, q\right)$. The image of $\sigma$ is the Segre variety $\Sigma_{r_{1} ; r_{2} ; \ldots ; r_{t}}$ : it can be regarded, in some way, as a product of projective spaces; see [15, Chap. 25], [13, Chap. 9] and [12, Chap. 2]. In the language of tensor products, $\sigma$ is the natural morphism between the varieties

$$
\mathrm{PG}\left(V_{1}, q\right) \times \mathrm{PG}\left(V_{2}, q\right) \times \cdots \times \mathrm{PG}\left(V_{t}, q\right) \longrightarrow \mathrm{PG}\left(V_{1} \otimes V_{2} \otimes \cdots \otimes V_{t}, q\right) .
$$

In this paper, we are interested in the case $r_{1}=r_{2}=\ldots=r_{t}=r$; for brevity we shall write $\Sigma_{r^{t}}$ instead of $\Sigma_{r_{1} ; r_{2} ; \ldots ; r_{t}}$. Clearly, $\Sigma_{r^{t}} \subseteq$ PG $\left(r^{t}-1, q\right)$.

Take now the projective space $\mathrm{PG}\left(r-1, q^{t}\right)$ and let $v \longmapsto v^{q}$ be the $\mathbb{F}_{q}$-linear collineation of order $t$ induced by the Frobenius automorphism of the extension $\left[\mathbb{F}_{q^{t}}\right.$ : $\left.\mathbb{F}_{q}\right]$. For any $P \in \mathrm{PG}\left(r-1, q^{t}\right)$, write

$$
P^{\alpha}=\sigma\left(\left[P, P^{q}, \ldots, P^{q^{t-1}}\right]\right) .
$$

The image of this correspondence is the variety $\mathcal{V}_{r, t}$. It is immediate to see that the $\mathbb{F}_{q}$-linear collineation of order $t$ given by

$$
\left(p_{1} \otimes p_{2} \otimes \cdots \otimes p_{t}\right) \longmapsto\left(p_{t}^{q} \otimes p_{1}^{q} \otimes \cdots \otimes p_{t-1}^{q}\right)
$$

fixes $\mathcal{V}_{r, t}$ point-wise; hence, $\mathcal{V}_{r, t}$ is contained in a subgeometry $\Omega=\mathrm{PG}\left(r^{t}-1, q\right)$ of PG $\left(r^{t}-1, q^{t}\right)$. It turns out that $\mathcal{V}_{r, t}$ is, in fact, the complete intersection of the Segre product $\Sigma_{r^{t}}$ with $\Omega$.

As an algebraic variety $\mathcal{V}_{r, t}$ first appeared in [26]; it has then been described in [21] and therein extensively studied. Recently, in [25], an explicit parametrization for $\mathcal{V}_{r, t}$ has been determined, leading to the discovery of some new properties. It is convenient to recall here this parametrization. Let $\mathfrak{F}$ be the set of all functions $f:\{0, \ldots, t-1\} \rightarrow\{0, \ldots, r-1\}$ and write $P=\left(x_{0}, \ldots, x_{r-1}\right) \in \mathrm{PG}\left(r-1, q^{t}\right)$. Then, there is an injective map $\alpha$ : PG $\left(r-1, q^{t}\right) \rightarrow \mathcal{V}_{r, t} \subseteq \mathrm{PG}\left(r^{t}-1, q^{t}\right)$ sending any $P \in \mathrm{PG}\left(r-1, q^{t}\right)$ to the point $P^{\alpha} \in \mathrm{PG}\left(r^{t}-1, q^{t}\right)$ whose coordinates consist of all products of the form

$$
\prod_{i=0}^{t-1} x_{f(i)}^{q^{i}}
$$

as $f$ varies in $\mathfrak{F}$. 
We shall also make use of the alternative description of $\mathcal{V}_{r, t}$ from [21, 26]. A Desarguesian (also called normal) spread of $\mathrm{PG}(r t-1, q)$ is projectively equivalent to a linear representation of PG $\left(r-1, q^{t}\right)$ in PG $(r t-1, q)$; see [26]. As such, it consists of a collection $\mathcal{S}$ of $(t-1)$-dimensional subspaces of PG $(r t-1, q)$, each of them the linear representation of a point of PG $\left(r-1, q^{t}\right)$, partitioning the point set of PG $(r t-1, q)$. When regarded on the Grassmannian of all the $(t-1)$-dimensional subspaces of PG $(r t-1, q)$, the elements of $\mathcal{S}$ determine the algebraic variety $\mathcal{V}_{r, t}$. The best known example is for $r=t=2$ : indeed, the Grassmannian of the lines of a Desarguesian spread of PG $(3, q)$ is an elliptic quadric $\mathcal{V}_{2,2}=\mathcal{Q}^{-}(3, q)$; see, for instance, [14, Sect. 15.4].

More generally, if $\Pi_{P} \in \mathcal{S}$ is the linear representation of a point $P \in \mathrm{PG}(r-$ $\left.1, q^{t}\right)$, then the image under the Grassmann map of $\Pi_{P}$ is $P^{\alpha}$. Using this correspondence, it has been possible to investigate several properties of $\mathcal{V}_{r, t}$; see $[19,21$, 25]. Here we will recall just some of them. The group P PL $\left(r, q^{t}\right)$ preserves a Desarguesian $(t-1)$-spread $\mathcal{S}$ of PG $(r t-1, q)$, thus its lifting preserves $\mathcal{V}_{r, t}$ and its action on the points of $\mathcal{V}_{r, t}$ is isomorphic to the 2-transitive action of $\operatorname{P\Gamma L}\left(r, q^{t}\right)$ on the elements of $\mathcal{S}$; see [21]. We remark that the aforementioned action is actually 3-transitive for $r=2$.

The group $G:=\operatorname{PGL}\left(r, q^{t}\right)$ acts in a natural way on $M=\operatorname{PG}\left(r-1, q^{t}\right)$, which is both a $G$-module and an $\mathbb{F}_{q^{t}}$-vector space. The twisted tensor product has been introduced in [28] to realize a new $G$-module, say $M^{\prime}$, defined over the subfield $\mathbb{F}_{q}$ from $M$; this induces a straightforward embedding of $\operatorname{PGL}\left(r, q^{t}\right)$ in $\operatorname{PGL}\left(r^{t}, q\right)$. We briefly recall the construction. Write the action of $G$ on $M$ as $g \cdot P \rightarrow g P$, where $g \in G$ and $P \in M$. For any automorphism $\phi$ of $\mathbb{F}_{q^{t}}$, we can define a new $G$-module, $M^{\phi}$ with group action $g \cdot P \rightarrow g^{\phi} P$; when $\phi$ is the automorphism $g \rightarrow g^{q^{i}}$, we shall write $M^{\phi}=M^{q^{i}}$. Using this notation, the twisted tensor product of $M$ over $\mathbb{F}_{q}$ is

$$
M^{\prime}=M \otimes M^{q} \otimes \cdots \otimes M^{q^{t-1}} .
$$

If we restrict our attention to the points of $\mathcal{V}_{r, t}$, we see that for any $g \in G$ and $P \in$ $\mathrm{PG}\left(r-1, q^{t}\right)$

$$
(g P)^{\alpha}=\sigma\left(\left[g P, g^{q} P^{q}, \ldots, g^{q^{t-1}} P^{q^{t-1}}\right]\right)=g \sigma\left(\left[P, P^{q}, \ldots, P^{q^{t-1}}\right]\right)=g P^{\alpha} .
$$

This is to say that PGL $\left(r, q^{t}\right)$, as embedded in PGL $\left(r^{t}, q\right)$, it stabilizes $\mathcal{V}_{r, t}$ and its action on the points of the variety is the same way as that on those of PG $\left(r-1, q^{t}\right)$. For this reason, we can consider $\mathcal{V}_{r, t}$ as a geometric realization of the twisted tensor product group embedding; in brief we shall call it the twisted tensor embedding over $\mathbb{F}_{q}$ of PG $\left(r-1, q^{t}\right)$.

In this paper, we will consider the varieties $\mathcal{V}_{r, t}$ for $(r, t) \neq(2,2)$ and show how they behave with respect to suitable polarities.

\section{Desarguesian complete partial ovoids}

In this section, the constructions of $[4,5]$ are generalized to any $q$ and for $t \geq 4$. Our main result is Theorem 1 . 
The algebraic variety $\mathcal{V}_{2, t} \subset \mathrm{PG}\left(2^{t}-1, q\right)$ is the image under the Grassmann map of the elements of a Desarguesian $(t-1)$-spread $\mathcal{S}$ of PG $(2 t-1, q)$ and it has as automorphism group a lifting of PGL $\left(2, q^{t}\right)$, hence a group isomorphic to PGL $\left(2, q^{t}\right)$ and with the same 3 -transitive action. The sublines PG $(1, q)$ embedded in PG $\left(1, q^{t}\right)$ correspond to the reguli of $\mathcal{S}$ and their image under $\alpha$ are normal rational curves of degree $t$, intersection of $\mathcal{V}_{2, t}$ with suitable $t$-dimensional spaces; conversely, for $t<q$, every normal rational curve of degree $t$ is image of a subline, or a regulus; see [19]. Hence, via this correspondence, the points of $\mathcal{V}_{2, t}$ and the normal curves of degree $t$ contained in $\mathcal{V}_{2, t}$ form a $3-\left(q^{t}+1, q+1,1\right)$ design. Also, in [25] it has been proved that any $t+1$ points of $\mathcal{V}_{2, t}$ are in generic position, i.e. any $t+1$ of them span a $t$-dimensional space.

Throughout this section we assume $t \geq 3$. In [5], Cossidente and King prove with a different approach that if $q=2^{h}$, then the points of $\mathcal{V}_{2, t}$ form a partial ovoid of the hyperbolic quadric $\mathcal{Q}^{+}\left(2^{t}-1, q\right)$ and it is complete since its size attains the Blokhuis-Moorhouse bound of [1]. In [4], Cossidente proves that, for $q$ odd and $t=3$, the points of $\mathcal{V}_{2, t}$ form a partial ovoid with respect to a suitable symplectic polarity of $\operatorname{PG}(7, q)$. The aim of this section is to prove that for $t \geq 3$ the points of $\mathcal{V}_{2, t}$ form a partial ovoid with respect to a suitable polarity of $\operatorname{PG}\left(2^{t}-1, q\right)$, for both even and odd $q$.

By the parametrization given in [25], $\mathcal{V}_{2, t}$ is the image of the injective map $\alpha: P=$ $\left(x_{0}, x_{1}\right) \in \mathrm{PG}\left(1, q^{t}\right) \longmapsto P^{\alpha} \in \mathrm{PG}\left(2^{t}-1, q^{t}\right)$, where the coordinates of $P^{\alpha}$ consist of all products of the form

$$
\prod_{i=0}^{t-1} x_{f(i)}^{q^{i}}
$$

as $f$ varies in $\mathfrak{F}, \mathfrak{F}=\{f:\{0,1, \ldots, t-1\} \rightarrow\{0,1\}\}$. Let $S$ be the set $\{0,1, \ldots, t-1\}$ and $\mathcal{P}(S)$ be the power set of $S$, hence we have $\mathcal{V}_{2, t}=\left\{P_{\infty}\right\} \cup\left\{P_{x}, x \in \mathbb{F}_{q^{t}}\right\}$, where $P_{\infty}$ is the point $(0,0, \ldots, 0,1)$ and $P_{x}$ is the point whose coordinates are $\prod_{i \in T} x^{q^{i}}$, with $T \in \mathcal{P}(S)$, and the convention that when $T=\emptyset$ the corresponding coordinate is $1, \forall x \in \mathbb{F}_{q^{t}}$. We choose an ordering in $\mathcal{P}(S)$ such that the first element is the empty set and the complement of the $i$ th element of $\mathcal{P}(S)$ is the $\left(2^{t}-i+1\right)$ th element and from now on we denote by $T_{i}$ the $i$ th element of $\mathcal{P}(S)$ with respect to this ordering. We label the components of the coordinates vector of the points of $\mathcal{V}_{2, t} \backslash\left\{P_{\infty}\right\}$ according to this, i.e. the $i$ th component of the coordinates vector of $P_{x}$ is $\prod_{j \in T_{i}} x^{q^{j}}$. Let $\sigma$ be the permutation $(01 \cdots t-1)$ of $S$ and let $L_{\sigma}$ be the $\mathbb{F}_{q}$-linear collineation:

$$
\begin{aligned}
& \left(v_{1}, v_{2}, \ldots, v_{2^{t}}\right) \in \mathrm{PG}\left(2^{t}-1, q^{t}\right) \\
& \quad \rightarrow\left(v_{\sigma^{-1}(1)}^{q}, v_{\sigma^{-1}(2)}^{q}, \ldots, v_{\sigma^{-1}\left(2^{t}\right)}^{q}\right) \in \mathrm{PG}\left(2^{t}-1, q^{t}\right),
\end{aligned}
$$

where, by abuse of notation, we have denoted by $\sigma^{-1}(i)$ the map $i \rightarrow j \Longleftrightarrow T_{i}^{\sigma^{-1}}=$ $T_{j}, T_{i}$ and $T_{j} \in \mathcal{P}(S)$. As the largest orbit under the action of $\sigma$ on $\mathcal{P}(S)$ has order $t, L_{\sigma}$ is an $\mathbb{F}_{q}$-linear collineation of order $t$ of $\mathrm{PG}\left(2^{t}-1, q^{t}\right)$, hence the set of its fixed points is a subgeometry PG $\left(2^{t}-1, q\right)$. Let $\mathcal{R}$ be a set of representatives for the orbits of $\mathcal{P}(S)$ under the action of $\sigma$ and let $T_{i} \in \mathcal{R}$. If the orbit of $T_{i}$ under the action of $\sigma$ has order $s, s \mid t$, and $\left\{T_{i}, T_{i}^{\sigma}, \ldots, T_{i}^{\sigma^{s-1}}\right\}=\left\{T_{i}, T_{i_{1}}, \ldots, T_{i_{s-1}}\right\}$ in the chosen 
ordering, then the components $v_{i}, v_{i_{1}}, \ldots, v_{i_{s-1}}$ of the point $P=\left(v_{1}, v_{2}, \ldots, v_{2^{t}}\right)$ of the subgeometry PG $\left(2^{t}-1, q\right) \subset \mathrm{PG}\left(2^{t}-1, q^{t}\right)$ are such that $v_{i} \in \mathbb{F}_{q^{s}}$ and $v_{i_{j}}=v_{i}^{q^{j}}$, $\forall j=1, \ldots, s-1$. By the relation: $\left(\prod_{i \in T} x^{q^{i}}\right)^{q}=\prod_{i \in T^{\sigma}} x^{q^{i}}$, we find that $\mathcal{V}_{2, t}$ is fixed point-wise by $L_{\sigma}$ and so the subgeometry $\mathrm{PG}\left(2^{t}-1, q\right)$ where $\mathcal{V}_{2, t}$ in fact lies is the one defined by $L_{\sigma}$.

In $\mathrm{PG}\left(2^{t}-1, q^{t}\right)$ we consider the following non-degenerate bilinear form: $\beta(u, v)=\sum_{i=1}^{2^{t}} \epsilon_{i} u_{i} v_{2^{t}-i+1}$, where $u=\left(u_{1}, u_{2}, \ldots, u_{2^{t}}\right), v=\left(v_{1}, v_{2}, \ldots, v_{2^{t}}\right) \in$ PG $\left(2^{t}-1, q^{t}\right), \epsilon_{i}=(-1)^{\left|T_{i}\right|}$ and $T_{i} \in \mathcal{P}(S)$. When $t$ is even, the parity of $\left|T_{i}\right|$ and $\left|S \backslash T_{i}\right|$ are the same, so the form $\beta$ is symmetric and the induced polarity is orthogonal; conversely, when $t$ is odd, then $\beta$ is alternating and the induced polarity is symplectic. We want to describe the polarity induced by $\beta$ on the subgeometry PG $\left(2^{t}-1, q\right)$. We observe that if the $\sigma$-orbit of $T_{i}$ has size $s$, then also the $\sigma$-orbit of $T_{i}^{c}:=S \backslash T_{i}$ does, since it easy to see that $T_{i}^{\sigma c}=T_{i}^{c \sigma}$. As a consequence, $v_{i} \in \mathbb{F}_{q^{s}}$ if and only if $v_{2^{t}-i+1} \in \mathbb{F}_{q^{s}}, \forall v=\left(v_{1}, v_{2}, \ldots, v_{2^{t}}\right) \in \operatorname{PG}\left(2^{t}-1, q\right)$. Let $\operatorname{Tr}^{(s)}: x \in \mathbb{F}_{q^{s}} \mapsto x+x^{q}+\cdots+x^{q^{s-1}} \in \mathbb{F}_{q}$ be the trace function $\forall s \mid t$. Now, by abuse of notation we denote by $\beta$ also the bilinear form induced on the subgeometry under consideration and so we have $\beta(u, v)=\sum_{i \mid T_{i} \in \mathcal{R}} \epsilon_{i} \operatorname{Tr}^{s_{i}}\left(u_{i} v_{2^{t}-i+1}\right)$, with $u_{i} \in \mathbb{F}_{q^{s_{i}}}, s_{i} \mid t$, and $u=\left(u_{1}, u_{2}, \ldots, u_{2^{t}}\right), v=\left(v_{1}, v_{2}, \ldots, v_{2^{t}}\right) \in \operatorname{PG}\left(2^{t}-1, q\right)$. Again, it is easy to see that $\beta$ is non-degenerate and symmetric if $t$ is even, alternating if $t$ is odd.

When $t$ is odd we have a symplectic polarity in $\mathrm{PG}\left(2^{t}-1, q\right)$ and for even $q$ we wish to determine a suitable quadratic form inducing it. As in this case $T_{i}$ and $T_{i}^{c}$ have different size, they give rise to two different orbits under the action of $\sigma, \forall T_{i} \in \mathcal{P}(S)$; thus the number of $\sigma$-orbits is even and we denote by $\mathcal{R}^{\prime}$ the subset of $\mathcal{R}$ consisting of the elements of size at most $\frac{t-1}{2}$; by the previous observation, we have $\left|\mathcal{R}^{\prime}\right|=$ $|\mathcal{R}| / 2$. Then, when $q$ is even, the quadric $\mathcal{Q}$ of equation $\sum_{i \mid T_{i} \in \mathcal{R}^{\prime}} \operatorname{Tr}^{s_{i}}\left(u_{i} u_{2^{t}-i+1}\right)=0$ polarizes $\beta$ and so induces the symplectic polarity. The bilinear function $(a, b) \in$ $\mathbb{F}_{q^{s}} \times \mathbb{F}_{q^{s}} \mapsto \operatorname{Tr}^{s}(a b) \in \mathbb{F}_{q}$ is obviously a scalar product, so by its equation, we can easily see that $\mathcal{Q}=\mathcal{Q}^{+}\left(2^{t}-1, q\right)$. The point $P_{\infty} \in \mathcal{V}_{2, t}$ clearly belongs to $\mathcal{Q}$; the point $P_{x}=\left(u_{1}, u_{2}, \ldots, u_{2^{t}}\right) \in \mathcal{V}_{2, t}$ is such that $u_{i}=\prod_{j \in T_{i}} x^{q^{j}}$, so if we denote by $\mathrm{N}$ the norm function: $x \in \mathbb{F}_{q^{t}} \mapsto \mathrm{N}(x)=x^{1+q+\cdots+q^{t-1}} \in \mathbb{F}_{q}$, then $u_{i} u_{2^{t}-i+1}=\mathrm{N}(x)$, $\forall i=1,2, \ldots, 2^{t}$. If we plug in the coordinates of a point $P_{x}$ in the equation of $\mathcal{Q}$, we obtain $\sum_{i=1}^{2^{t-1}} \mathrm{~N}(x)=\mathrm{N}(x) 2^{t-1}=0, \forall x \in \mathbb{F}_{q^{t}}$, because the characteristic is even. So for $t$ odd and $q$ even, we have determined a hyperbolic quadric $\mathcal{Q}=\mathcal{Q}^{+}\left(2^{t}-1, q\right)$ polarizing the alternating form $\beta$ (and hence inducing the symplectic polarity) and containing the points of $\mathcal{V}_{2, t}$.

Suppose now $t$ to be even. The form $\beta$ is orthogonal, hence it is induced by a quadric $\mathcal{Q}$. We want to prove that such a quadric is hyperbolic. Let $\mathcal{R}^{*}$ be the subset of $\mathcal{R}$ such that $T_{i}$ and $T_{i}^{c}$ are not in the same orbit under the action of $\sigma$. We observe that $\left|\mathcal{R}^{*}\right|$ is even, as the $\sigma$-orbits of $T_{i}$ and $T_{i}^{c}$ are distinct, $\forall T_{i} \in \mathcal{R}^{*}$. Let $\mathcal{R}^{* \prime}$ be the subset of $\mathcal{R}^{*}$ such that $\left|T_{i}\right| \leq t / 2$ and, once more, we note that $\left|\mathcal{R}^{* \prime}\right|=\left|\mathcal{R}^{*}\right| / 2$. Consider the subspace $\Pi_{1}$ of PG $\left(2^{t}-1, q\right)$ defined by the equations $u_{i}=0$, for all $i$ such that $T_{i}$ and $T_{i}^{c}$ are in the same $\sigma$-orbit, then $\Pi_{1}$ has odd dimension and is met by $\mathcal{Q}$ in a quadric $\mathcal{Q}_{1}$ of equation $\sum_{i \mid T_{i} \in \mathcal{R}^{*}} \epsilon_{i} \operatorname{Tr}^{s_{i}}\left(u_{i} u_{2^{t}-i+1}\right)=0$, that, as before, is hyperbolic. Let now $\mathcal{R}^{* *}$ be the subset of $\mathcal{R}$ such that $T_{i}$ and $T_{i}^{c}$ are in the same 
orbit under the action of $\sigma$, i.e., $T_{i}^{c}=T_{i}^{\sigma^{s_{i}}}$, so the $\sigma$-orbit of $T_{i}$ has size $2 s_{i}, 2 s_{i} \mid t$ and $u_{i} \in \mathbb{F}_{q^{2 s_{i}}}$. The subspace $\Pi_{2}$ of PG $\left(2^{t}-1, q\right)$ defined by the equations $u_{i}=0$ for all $i$ such that $T_{i}$ and $T_{i}^{c}$ are not in the same $\sigma$-orbit is skew with $\Pi_{1}$ and so it also has odd dimension; furthermore, the intersection of $\mathcal{Q}$ with $\Pi_{2}$ is a quadric $\mathcal{Q}_{2}$ of equation $\sum_{i \mid T_{i} \in \mathcal{R}^{* *}} \epsilon_{i} \operatorname{Tr}^{s_{i}}\left(u_{i}^{1+q^{s_{i}}}\right)=0$. The quadratic equation $\operatorname{Tr}^{s}\left(u^{1+q^{s}}\right)=0$, $u \in \mathbb{F}_{q^{2 s}}$, is non-degenerate, hence the quadric is either elliptic or hyperbolic in a $(2 s-1)$-projective space. Since the number of (projective) solutions of such an equation is $\left(q^{s}-1\right)\left(q^{s}+1\right) /(q-1)$, it is immediate to see that this corresponds to an elliptic quadric (for the size of a quadric in a projective space, see e.g. [15, Theorem 22.5.1(b)]). The quadric $\mathcal{Q}_{2}$ is hence the orthogonal sum of elliptic quadrics, so to determine its nature, it is necessary to look at the parity of the number of the elliptic quadrics occurring in such a sum. Following the terminology of [5], an element $T \in \mathcal{P}(S)$ of size $t / 2$ such that the $\sigma$-orbit of $T$ has size $r$ and such that $T$ and $T^{c}$ are in the same orbit is an invertible pattern of irreducible length $r$ and the number of these elements is denoted by $a(r)$. By [5, Lemma 3.2(4)], $\sum_{r \mid t} a(r) / r$ is even, implying, going back to our terminology, that $\left|\mathcal{R}^{* *}\right|$ is even. We can conclude that $\mathcal{Q}_{2}$ is the orthogonal sum of an even number of elliptic quadrics, hence it is hyperbolic. Finally, since $\mathcal{Q}$ is the orthogonal sum of the two hyperbolic quadrics $\mathcal{Q}_{1}$ and $\mathcal{Q}_{2}, \mathcal{Q}$ is hyperbolic as well, i.e. $\mathcal{Q}=\mathcal{Q}^{+}\left(2^{t}-1, q\right)$. As before, the point $P_{\infty} \in \mathcal{V}_{2, t}$ clearly belongs to $\mathcal{Q}$ and $P_{x}=\left(u_{1}, u_{2}, \ldots, u_{2^{t}}\right) \in \mathcal{V}_{2, t}$ is such that $u_{i} u_{2^{t}-i+1}=\mathrm{N}(x)$, $\forall i=1,2, \ldots, 2^{t}$, so if we plug in the coordinates of the point $P_{x}$ in the equation of $\mathcal{Q}$ we obtain $\sum_{i=1}^{2^{t-1}} \epsilon_{i} \mathrm{~N}(x)=\mathrm{N}(x) \sum_{i=1}^{2^{t-1}} \epsilon_{i}=\mathrm{N}(x)(n-m)$, where $2 n$ is the number of subsets of $S$ of even size and $2 m$ is the number of subsets of $S$ of odd size. In the vector space $V(t, 2)$, we can identify each element of $\mathcal{P}(S)$ with its characteristic vector, and the elements of even size are those whose characteristic vector satisfies the equation $v_{1}+v_{2}+\cdots+v_{t}=0$, hence there are $2^{t-1}$ such, so $2 n=2 m=2^{t-1}$. We can conclude that $\mathrm{N}(x)(n-m)=0 \forall x \in \mathbb{F}_{q^{t}}$. Hence, also in this case, the variety $\mathcal{V}_{2, t}$ is completely contained in the quadric $\mathcal{Q}=\mathcal{Q}^{+}\left(2^{t}-1, q\right)$.

For all $t \geq 3$ and $q$, let $\perp$ be the polarity induced by $\beta$ on $\mathrm{PG}\left(2^{t}-1, q\right)$. The hyperplane $P_{\infty}^{\perp}$ has equation $u_{1}=0$, hence $P_{x} \notin P_{\infty}^{\perp} \forall x \in \mathbb{F}_{q^{t}}$. It is easy to see that $P_{x} \in P_{y}^{\perp}$ if and only if $\mathrm{N}(x-y)=0$ and this is possible if and only if $x=y$. So we have $P_{x} \notin P_{y}^{\perp} \forall x \neq y$ and so the points of $\mathcal{V}_{2, t}$ form a partial ovoid with respect to $\perp$.

Finally, the last result we want to prove is that $\mathcal{V}_{2, t}$ is complete, i.e. for any $R \notin \mathcal{V}_{2, t}$ in the polar space we have $R^{\perp} \cap \mathcal{V}_{2, t} \neq \emptyset$. A hyperplane section of $\mathcal{V}_{2, t}$ is represented by an $\mathbb{F}_{q}$-linear homogeneous equation in $\prod_{i \in T, j \in T^{c}} x^{q^{i}} y^{q^{j}}$, as $T$ varies in $\mathcal{P}(S)$, with $x, y \in \mathbb{F}_{q^{t}}$. Working out this equation in $\mathbb{F}_{q}$, we get a homogeneous equation of degree $t$ with $2 t$ variables, which by the Chevalley-Warning Theorem has $p \lambda$ solutions, where $p$ is the characteristic of the field; in particular this equation has always at least a projective solution, and so every hyperplane intersects $\mathcal{V}_{2, t}$ in at least one point. It follows that the partial ovoid is complete.

We summarize the results proven in this section in the following theorem.

Theorem 1 Let $\mathbb{P}$ be the polar space induced by a $\mathcal{Q}^{+}\left(2^{t}-1, q\right)$ if $t$ is even or $t$ is odd and $q$ is even and let $\mathbb{P}$ be the symplectic polar space of $\mathrm{PG}\left(2^{t}-1, q\right)$ if 
both $t$ and $q$ are odd. The points of $\mathcal{V}_{2, t}$ form a complete partial ovoid of $\mathbb{P}$ with the following properties:

- the size is $q^{t}+1$;

- the stabilizer is isomorphic to PGL $\left(2, q^{t}\right)$ with a 3-transitive action on the points;

- any $t+1$ points are in general position;

- for $t<q$, the points and the normal rational curves of degree $t$ contained in it form a 3- $\left(q^{t}+1, q+1,1\right)$ design.

Remark 1 For $t=3$, we obtain the so called Desarguesian ovoid of $\mathcal{Q}^{+}(7, q), q$ even (see [16]).

\section{Unitary complete partial ovoids of quadrics}

In this section we consider the variety $\mathcal{V}_{r, 2}$ of $\mathrm{PG}\left(r^{2}-1, q\right), r \geq 3, q>2$ : it is the twisted tensor embedding of PG $\left(r-1, q^{2}\right)$ over $\mathbb{F}_{q}$ or, in another terminology, it is the intersection of the Segre product of two PG $\left(r-1, q^{2}\right)$ with a Baer subgeometry $\mathrm{PG}\left(r^{2}-1, q\right)$. As we are considering in this case a 2 -dimensional tensor, there is a natural representation of this space by means of matrices. More precisely, as $r^{2}$ dimensional vector space over $\mathbb{F}_{q^{2}}$, we consider the set $\mathcal{M}$ of square matrices of order $r$ over $\mathbb{F}_{q^{2}}$. In the projective space PG $\left(r^{2}-1, q^{2}\right)$ defined by $\mathcal{M}$ consider the $\mathbb{F}_{q}$-linear collineation of order two $\sigma: M \in \mathcal{M} \mapsto M^{T q} \in \mathcal{M}$, where $M^{T}$ is the transpose of $M$ and $M^{q}$ is the matrix obtained from $M$ by raising every component of $M$ to the $q$ th power. The set of points fixed by $\sigma$ is a subgeometry $\mathrm{PG}\left(r^{2}-1, q\right)$ and it consists of the so called Hermitian matrices of order $r$, i.e. the matrices $M \in \mathcal{M}$ such $M^{T q}=M$. Then, the variety $\mathcal{V}_{r, 2}$ is the image of the map

$$
\alpha: \quad\left(x_{1}, x_{2}, \ldots, x_{r}\right) \in \mathrm{PG}\left(r-1, q^{2}\right) \mapsto M \in \mathrm{PG}\left(r^{2}-1, q\right) \mid m_{i j}=x_{i} x_{j}^{q} .
$$

This variety has already been studied in detail in [7] for $r=3$ and in [3] for general $r$, and in the literature it is usually called the Hermitian Veronesean. An important property of the Hermitian Veronesean $\mathcal{V}_{r, 2}$ we will use in this section is that every hyperplane section of $\mathcal{V}_{r, 2}$ is the image under $\alpha$ of a (possibly singular) Hermitian variety of $\mathrm{PG}\left(r-1, q^{2}\right)$.

Define on $\mathcal{M}$ the following quadratic form: $Q(M)=\sum_{i<j} m_{i i} m_{j j}-$ $\sum_{i<j} m_{i j} m_{j i}$, that is, the sum of the principal minors of order two of $M$. On PG $\left(r^{2}-1, q\right), Q$ induces the following quadratic form, denoted, by abuse of notation, with $Q$ as well: $Q(M)=\sum_{i<j} m_{i i} m_{j j}-\sum_{i<j} m_{i j}^{q+1}$. If $M \in \mathcal{V}_{r, 2}$, then, clearly, $Q(M)=0$. Let $\operatorname{Tr}(M)=\sum_{i=1}^{r} m_{i i}$ be the trace of the matrix $M$; as $\operatorname{Tr}(M)=0$ defines a hyperplane of $\mathcal{M}$ that it is fixed by $\sigma$, it also determines a hyperplane $H$ of $\mathrm{PG}\left(r^{2}-1, q\right)$. The points of $H \cap \mathcal{V}_{r, 2}$ are the images under $\alpha$ of the points of the non-singular Hermitian variety $\mathcal{H}\left(r-1, q^{2}\right)$ of equation $\sum_{i=1}^{r} x_{i}^{q+1}=0$. On the hyperplane $H$, the quadratic form $Q$ induces the form $Q^{\prime}(M)=\sum_{i=1}^{r-1} m_{i i}^{2}+\sum_{i<j<r} m_{i i} m_{j j}+\sum_{i<j} m_{i j}^{q+1}$ and hence the symmetric bilinear form $\beta(M, N)=\operatorname{Tr}(M N)$, where by $M N$ we have denoted the usual matrix product. Let $\mathbf{x} \cdot \mathbf{y}:=\sum_{i=1}^{r} x_{i} y_{i}$ for all $\mathbf{x}=\left(x_{1}, x_{2}, \ldots, x_{r}\right)$ and $\mathbf{y}=$ 
$\left(y_{1}, y_{2}, \ldots, y_{r}\right) \in \mathrm{PG}\left(r-1, q^{2}\right)$, and let $\mathbf{x}^{q}$ be $\left(x_{1}^{q}, x_{2}^{q}, \ldots, x_{r}^{q}\right)$, then the polar hyperplane of $P=\left(u_{1}, u_{2}, \ldots, u_{r}\right) \in \mathrm{PG}\left(r-1, q^{2}\right)$ with respect the Hermitian polarity induced by $\mathcal{H}\left(r-1, q^{2}\right)$ has equation $\mathbf{u}^{q} \cdot \mathbf{x}=0$. Write $M(\mathbf{x}):=\mathbf{x}^{\alpha}$, then two points $M(\mathbf{x})$ and $M(\mathbf{y})$ of $\mathcal{V}_{r, 2} \cap H$ are collinear with respect to $\beta$ if and only if $\operatorname{Tr}(M(\mathbf{x}) M(\mathbf{y}))=0$, but by straightforward computation, we have $\operatorname{Tr}(M(\mathbf{x}) M(\mathbf{y}))=$ $\left(\sum_{j=1}^{r} x_{j} y_{j}^{q}\right)\left(\sum_{i=1}^{r} x_{i}^{q} y_{i}^{q}\right)=\left(\mathbf{x} \cdot \mathbf{y}^{q}\right)\left(\mathbf{x}^{q} \cdot \mathbf{y}\right)$, hence $M(\mathbf{x})$ and $M(\mathbf{y})$ are collinear with respect to $\beta$ if and only if the points $\mathbf{x}$ and $\mathbf{y}$ of $\mathrm{PG}\left(r-1, q^{2}\right)$ are collinear in $\mathcal{H}\left(r-1, q^{2}\right)$. Our aim is to construct large (complete) partial ovoids of $\mathrm{PG}\left(r^{2}-2, q\right)$ with respect the orthogonal polarity defined by $Q^{\prime}$ and contained in the algebraic variety $\mathcal{V}_{r, 2}$ that are image of ovoids of $\mathcal{H}\left(r-1, q^{2}\right)$. We first prove the following result.

Theorem 2 In the PG $\left(r^{2}-2, q\right)$ defined by the Hermitian matrices of order $r$ and trace 0 , the bilinear form $\beta(M, N)=\operatorname{Tr}(M N)$ is degenerate if and only if $p \mid r$, where $p$ is the characteristic of $\mathbb{F}_{q}$. As a consequence, for $p \nmid r$, we see that the twisted tensor product of $\mathrm{PGU}(r, q)$ over $\mathbb{F}_{q}$ is embedded in the orthogonal group $\operatorname{PGO}\left(r^{2}-1, q\right)$ for $r$ even, and in the orthogonal group $\mathrm{PGO}^{\epsilon}\left(r^{2}-1, q\right)$ for $r$ odd, where $\epsilon$ has to be chosen in the following way:

- if $q$ is even and

$r \equiv 1 \bmod 8$, then $\epsilon=+$;

$r \equiv-1 \bmod 8$, then $\epsilon=-$;

$r \equiv 3 \bmod 8$, then $\epsilon=+$ when $\log _{2} q$ is odd and $\epsilon=-$ when $\log _{2} q$ is even;

$r \equiv-3 \bmod 8$, then $\epsilon=+$ when $\log _{2} q$ is even and $\epsilon=-$ when $\log _{2} q$ is odd;

- if $q$ is odd and

$r \equiv 1 \bmod 4$, then $\epsilon=+$ when $r$ is a square in $\mathbb{F}_{q}$ and $\epsilon=-$ when $r$ is a nonsquare in $\mathbb{F}_{q}$;

$r \equiv-1 \bmod 4$, then $\epsilon=-$ when $-r$ is a square in $\mathbb{F}_{q}$ and $\epsilon=+$ when $-r$ is a non-square in $\mathbb{F}_{q}$.

Finally, under the twisted tensor embedding over $\mathbb{F}_{q}$, pairwise non-collinear points of $\mathcal{H}\left(r-1, q^{2}\right)$ are mapped on pairwise non-collinear points of $\mathrm{PG}\left(r^{2}-2, q\right)$ with respect to the bilinear form $\beta$.

Proof The last part of the statement follows from the considerations preceding it. The radical of $\beta$ is $\left\{M \in \mathrm{PG}\left(r^{2}-2, q\right) \mid \operatorname{Tr}(M N)=0 \forall N \in \mathrm{PG}\left(r^{2}-2, q\right)\right\}$. Let $A_{i j}$ be the matrix with $a_{i j}=a_{j i}=1$ and all the other components 0 , and $B_{i j}$ the matrix with $b_{i j}=\xi, b_{j i}=\xi^{q}$ and all the other components $0, \xi \in \mathbb{F}_{q^{2}} \backslash \mathbb{F}_{q}, i<j$. Then, $\operatorname{Tr}\left(M A_{i j}\right)=m_{i j}+m_{i j}^{q}=0$ and $\operatorname{Tr}\left(M B_{i j}\right)=m_{i j} \xi+m_{i j}^{q} \xi^{q}=0$, hence $m_{i j}=0$ for all $i \neq j$. Let $C_{i}$ be the diagonal matrix with $c_{11}=1, c_{i i}=-1$ and $c_{j j}=0$ for $j \neq i, 1$, $i=2, \ldots, r$, then $\operatorname{Tr}\left(M C_{i}\right)=m_{11}-m_{i i}=0$ for all $i>1$. So $M=h I$, with $h \in \mathbb{F}_{q}$ and $I$ is the identity matrix. The matrix $I$ has trace 0 if and only if $p \mid r$, hence, in this case, $I$ belongs to the radical of $\beta$.

By the arguments in the previous paragraph and since $\alpha$ is the geometric realization of the twisted tensor product embedding of the groups, we find that the twisted tensor product of PGU $(r, q)$ is embedded in the orthogonal group related to 
the quadric $\mathcal{Q}$ of PG $\left(r^{2}-2, q\right)$ defined by $Q^{\prime}$, when $\mathcal{Q}$ is non-degenerate. It remains to determine, when $r$ is odd, whether $\mathcal{Q}$ is hyperbolic or elliptic. For every fixed pair $(i, j), i<j$, the line defined by the equations $m_{h k}=0 \forall(h, k) \neq(i, j)$ is non-isotropic with respect to $\mathcal{Q}$. The intersection of $\mathcal{Q}$ with the $(r-2)$-subspace of PG $\left(r^{2}-2, q\right)$ defined by the diagonal matrices with trace 0 is the quadric $\mathcal{Q}^{*}$ of equation $\sum_{i=1}^{r-1} m_{i i}^{2}+\sum_{i<j<r} m_{i i} m_{j j}=0$. We will determine its type using the invariant of [15, Theorem 22.2.1]. Suppose that the equation of a quadric of $\mathrm{PG}(n-1, q)$ is $\sum_{i=1}^{n} a_{i} x_{i}^{2}+\sum_{1 \leq i<j \leq n} a_{i j} x_{i} x_{j}=0$ with $n$ even and let $A=\left(a_{i j}\right)$ be such that $a_{i j}=a_{j i}, i<j$ and $a_{i i}=2 a_{i} \forall i=1, \ldots, n$ and $B$ be the matrix with $b_{i j}=-b_{j i}=a_{i j}, i<j$, and $b_{i i}=0 \forall i=1, \ldots, n$. In our case, $n=r-1$, $A=I+J$, where $I$ is the identity matrix and $J$ is the all 1 's matrix, and $B$ is the skew-symmetric matrix with $b_{i j}=1$ for all $1 \leq i<j \leq n$. In order to compute the invariant, we have to compute the determinant of $A$ and $B$. The eigenvalues of $J$ are 0 , with multiplicity $n-1$, and $n$, with multiplicity 1 , hence the eigenvalues of $A=I+J$ are 1 , with multiplicity $n-1$, and $n+1$, with multiplicity 1 , so $\operatorname{det} A=n+1=r$. In order to compute $\operatorname{det} B$, we use the theorem of [23, p. 397], which states that the determinant of a skew-symmetric matrix of even order is not altered by adding the same number to each component, so if we add 1 to all the components of $B$, we get a upper triangular matrix with all 1's on the diagonal, hence $\operatorname{det} B=1$. Let $\delta:=\frac{\operatorname{det} B-(-1)^{\frac{r-1}{2}} \operatorname{det} A}{4 \operatorname{det} B}=\frac{1-(-1)^{\frac{r-1}{2}} r}{4}$ if $q$ is even, and $\delta:=(-1)^{\frac{r-1}{2}} \operatorname{det} A=(-1)^{\frac{r-1}{2}} r$, if $q$ is odd. By [15, Theorem 22.2.1], if $q$ is even, then the quadric is hyperbolic or elliptic according to whether $t^{2}+t+\delta=0$ has two or no solutions; in our case $\delta=0$ if $r \equiv \pm 1 \bmod 8$ and $\delta=1$ if $r \equiv \pm 3 \bmod 8$, so the quadric $\mathcal{Q}^{*}$ is hyperbolic if $r \equiv \pm 1 \bmod 8$ or $r \equiv \pm 3 \bmod 8$ and $q=2^{2 h}$, whereas $\mathcal{Q}^{*}$ is elliptic if $r \equiv \pm 3 \bmod 8$ and $q=2^{2 h+1}$. If $q$ is odd, then the quadric is hyperbolic or elliptic according to whether $\delta$ is a non-zero square or a non-square, hence $\mathcal{Q}^{*}$ is hyperbolic or elliptic according to whether $(-1)^{\frac{r-1}{2}} r$ is a non-zero square or a non-square in $\mathbb{F}_{q}$. The polar space induced by the quadric $\mathcal{Q}$ is the orthogonal sum of $\frac{r^{2}-r}{2}$ non-isotropic lines and the polar space induced by $\mathcal{Q}^{*}$. The orthogonal sum of $\frac{r^{2}-r}{2}$ non-isotropic lines is a polar space of hyperbolic type if $r \equiv 1 \bmod 4$ and it is of elliptic type if $r \equiv 3 \bmod 4$. Hence $\mathcal{Q}$ is hyperbolic or elliptic as described in the statement.

\section{1 (Complete partial) ovoids contained in $\mathcal{V}_{3,2}$}

If $r=3$, then the points of $\mathcal{H}\left(2, q^{2}\right)$ are pairwise non-collinear, since the Hermitian curve does not contain lines. The image under $\alpha$ of the points of $\mathcal{H}\left(2, q^{2}\right)$ is a set of non-collinear points of the quadric $\mathcal{Q}$ of $\mathrm{PG}(7, q)$ defined by $Q^{\prime}(M)=\sum_{i=1}^{2} m_{i i}^{2}+$ $\sum_{i<j} m_{i i} m_{j j}+\sum_{i<j} m_{i j}^{q+1}=0$. By Theorem 2 , the quadric $\mathcal{Q}$ is a cone with vertex the identity matrix for $q=3^{h} ; \mathcal{Q}$ is hyperbolic if $q=2^{2 h+1}$ or $q$ is odd and -3 is a non-zero square of $\mathbb{F}_{q}$, that is, $q \equiv 2 \bmod 3$, and, finally, $\mathcal{Q}$ is elliptic if $q=2^{2 h}$ or $q$ is odd and -3 is a non-zero square of $\mathbb{F}_{q}$, which is equivalent to say that $q \equiv 1 \bmod$ 3. We remark that this construction is the same as the one presented in [17, Sect. 6]. Hence, if $q \equiv 2 \bmod 3$, then $\mathcal{H}\left(2, q^{2}\right)^{\alpha}$ is the so called Unitary ovoid of $\mathcal{Q}^{+}(7, q)$ 
(see $[16,17,35])$, whereas if $q=3^{h}$, then we can project $\mathcal{H}\left(2, q^{2}\right)^{\alpha}$ from the vertex $I$ on the base of $\mathcal{Q}$, getting the ovoid of $\mathcal{Q}(6, q)$ appearing in $[16,17]$.

If $q \equiv 1 \bmod 3$, then the quadric obtained is a $\mathcal{Q}^{-}(7, q)$ and it is well known that elliptic quadrics of PG $(2 n+1, q), n>1$, do not admit ovoids (see [32]). Indeed, we get $q^{3}+1$ pairwise non-collinear points, whereas the ovoid number of $\mathcal{Q}^{-}(7, q)$ is $q^{4}+1$, hence we obtain a partial ovoid. Suppose that $\mathcal{H}\left(2, q^{2}\right)^{\alpha}$ is not complete, then there exists a point $P \in \mathcal{Q}^{-}(7, q)$ such that the polar hyperplane of $P$ has empty intersection with $\mathcal{H}\left(2, q^{2}\right)^{\alpha}$. So there exists a hyperplane $H^{\prime}$ of the PG $(8, q)$ where $\mathcal{V}_{3,2}$ lies such that $H^{\prime} \cap H \cap \mathcal{V}_{3,2}=\emptyset$, where $H$ is the hyperplane such that $\mathcal{H}\left(2, q^{2}\right)^{\alpha}=H \cap \mathcal{V}_{3,2}$. As well as for $H, H^{\prime} \cap \mathcal{V}_{3,2}$ is the image under $\alpha$ of a (possibly singular) Hermitian curve of $\operatorname{PG}\left(2, q^{2}\right)$, say $\mathcal{H}^{\prime}$. If $H^{\prime} \cap H \cap \mathcal{V}_{3,2}=\mathcal{H}\left(2, q^{2}\right)^{\alpha} \cap \mathcal{H}^{\prime \alpha}=\emptyset$, then we would find that two Hermitian curves, one of them non-singular, have empty intersection, but this is impossible (see [18] for the possible intersections of two Hermitian curves, with at least one of the two nonsingular). Hence $\mathcal{H}\left(2, q^{2}\right)^{\alpha}$ is a complete partial ovoid of $\mathcal{Q}^{-}(7, q), q \equiv 1 \bmod 3$, with stabilizer $\operatorname{PGU}(3, q)$. The best upper bound in the literature for complete partial ovoids of $\mathcal{Q}^{-}(7, q)$ is the one of [8], namely $2+\frac{1}{2} \frac{\left(q^{3}-1\right)\left(q^{2}+q+2\right)}{q+1}$, which is obviously not sharp since it is not an integer. So far, up to our knowledge, $\mathcal{H}\left(2, q^{2}\right)^{\alpha}$ provides the largest example of complete partial ovoid of $\mathcal{Q}^{-}(7, q)$.

\subsection{Complete partial ovoids contained in $\mathcal{V}_{4,2}$}

If $r>3$ and odd, then it has been proven in [32] that $\mathcal{H}\left(r-1, q^{2}\right)$ does not have ovoids, whereas if $r>4$ and even, the existence of ovoids of $\mathcal{H}\left(r-1, q^{2}\right)$ is still an open problem.

In the rest of the section, we shall focus on the case $r=4$ : in $\mathcal{H}\left(3, q^{2}\right)$ there are plenty of examples of ovoids. The algebraic variety $\mathcal{V}_{4,2}$ lies in a PG $(15, q)$ defined by the Hermitian matrices of order 4 . The intersection of $\mathcal{V}_{4,2}$ with the hyperplane of equation $\operatorname{Tr}(M)=0$ is the image under $\alpha$ of the non-singular Hermitian surface $\mathcal{H}\left(3, q^{2}\right)$ of equation $x_{1}^{q+1}+x_{2}^{q+1}+x_{3}^{q+1}+x_{4}^{q+1}=0$. In the PG $(14, q)$ defined by $\operatorname{Tr}(M)=0$, we have the quadratic form $Q^{\prime}(M)=m_{11}^{2}+m_{22}^{2}+m_{33}^{2}+m_{11} m_{22}+$ $m_{11} m_{33}+m_{22} m_{33}+m_{12}^{q+1}+m_{13}^{q+1}+m_{14}^{q+1}+m_{23}^{q+1}+m_{24}^{q+1}+m_{34}^{q+1}$. By Theorem 2 , the quadric $\mathcal{Q}$ defined by $Q^{\prime}(M)=0$ is a $\mathcal{Q}(14, q)$ for $q$ odd, and it is a cone with vertex the identity matrix $I$ for $q$ even. Suppose $q$ to be even, then the hyperplane of equation $m_{11}=0$ does not contain $I$. By abuse of notation, denote by $\mathcal{Q}$ the base of the cone contained in that hyperplane. Hence $\mathcal{Q}$ has equation $m_{22}^{2}+m_{33}^{2}+$ $m_{22} m_{33}+m_{12}^{q+1}+m_{13}^{q+1}+m_{14}^{q+1}+m_{23}^{q+1}+m_{24}^{q+1}+m_{34}^{q+1}=0$. The six lines defined by the equations $m_{22}=m_{33}=m_{h k}=0 \forall(h, k) \neq(i, j)$, for any fixed $(i, j), i<j$, are all non-isotropic lines. The line of equations $m_{i j}=0 \forall i<j$ is non-isotropic if and only if the polynomial $m_{22}^{2}+m_{33}^{2}+m_{22} m_{33}$ is irreducible, hence if $q=2^{2 h+1}$. The quadric $\mathcal{Q}$ is the orthogonal sum of these seven lines, hence $\mathcal{Q}$ is a $\mathcal{Q}^{-}(13, q)$ if $q=2^{2 h+1}$ and it is a $\mathcal{Q}^{+}(13, q)$ if $q=2^{2 h}$.

Let $\mathcal{O}$ be an ovoid of $\mathcal{H}\left(3, q^{2}\right)$; we recall that $|\mathcal{O}|=q^{3}+1$. If $q$ is odd, then $\mathcal{O}^{\alpha}$ is a partial ovoid of $\mathcal{Q}(14, q)$; if $q=2^{2 h}$, then $\mathcal{O}^{\alpha}$ can be projected onto a partial ovoid of $\mathcal{Q}^{+}(13, q)$ and finally if $q=2^{2 h+1}$, then $\mathcal{O}^{\alpha}$ can be projected onto a partial ovoid 
of $\mathcal{Q}^{-}(13, q)$. A (partial) ovoid of a quadric $\mathcal{Q}$ is a partial ovoid for every quadric containing $\mathcal{Q}$, hence, for a given partial ovoid $\mathcal{O}^{\alpha}$, it is interesting to find the smallest quadric containing it, as $\mathcal{O}^{\alpha}$ may not span the whole of $\mathrm{PG}(14, q)$.

Let $\mathcal{O}$ be the intersection of $\mathcal{H}\left(3, q^{2}\right)$ with a non-singular plane, then $\mathcal{O}=$ $\mathcal{H}\left(2, q^{2}\right)$ is clearly an ovoid, called the classical ovoid of $\mathcal{H}\left(3, q^{2}\right)$. In this case, it is easy to see $\mathcal{O}^{\alpha}$ is just the construction contained in the $\mathcal{V}_{3,2}$.

The two families of ovoids we wish to investigate now are those obtained by derivation from the classical one and the translation ovoids.

Let $\mathcal{O}$ be an ovoid of $\mathcal{H}\left(3, q^{2}\right), \ell$ be a line intersecting $\mathcal{O}$ in $q+1$ points and $\ell^{\prime}$ be the polar line of $\ell$ with respect the polarity induced by $\mathcal{H}\left(3, q^{2}\right)$. Then $\ell$ is a $(q+1)$ secant to $\mathcal{H}\left(3, q^{2}\right), \ell^{\prime}$ is a secant as well and $\mathcal{O}^{\prime}=(\mathcal{O} \backslash \ell) \cup\left(\ell^{\prime} \cap \mathcal{H}\left(3, q^{2}\right)\right)$ is again an ovoid of $\mathcal{H}\left(3, q^{2}\right)$. The ovoid $\mathcal{O}^{\prime}$ is said to be obtained by derivation from $\mathcal{O}$ and this technique has been introduced in [33]. It is possible to iterate this procedure by replacing more than one $(q+1)$-secant to $\mathcal{O}$ by the points of its polar line contained in $\mathcal{H}\left(3, q^{2}\right)$, as long as the lines pairwise meet in a point not in $\mathcal{O}$ and this is called multiple derivation. In literature, there are ovoids obtained by (multiple) derivation from the classical and from non-classical ones. We are interested in the derivation of the classical one; thus let $\mathcal{O}=\mathcal{H}\left(2, q^{2}\right)$ and $\ell$ be a line intersecting it in the $q+1$ points of a subline $\ell_{0}$. We know that the dimension of $\left\langle\mathcal{O}^{\alpha}\right\rangle$ is 7 and by [25, Theorem 2 ], the image of a subline $\ell_{0}$ is a conic contained in a plane. If we consider the image of $\mathcal{O} \backslash \ell_{0}$, then it is still 7-dimensional, because every point $P$ of $\ell_{0}$ is contained in another subline, hence $P^{\alpha}$ is contained in conic that has $q$ points in $\left(\mathcal{O} \backslash \ell_{0}\right)^{\alpha}$, so it is in the span of $\left(\mathcal{O} \backslash \ell_{0}\right)^{\alpha}$. The subgroup of PGU $(4, q)$ fixing $\mathcal{H}\left(2, q^{2}\right)$ acts transitively on the sublines of $\mathcal{H}\left(2, q^{2}\right)$, hence, we can assume without loss of generality that the $\mathcal{H}\left(2, q^{2}\right)$ under consideration is the one in the plane of equation $x_{4}=0$ and that the subline $\ell_{0}$ is $\left\{\left(x_{0}, x_{1}, 0,0\right), x_{0}^{q+1}+x_{1}^{q+1}=0\right\}$. The image of $\mathcal{H}\left(2, q^{2}\right)$ is contained in the space

$$
\left\{\left(\begin{array}{cccc}
m_{11} & m_{12} & m_{13} & 0 \\
m_{12}^{q} & m_{22} & m_{23} & 0 \\
m_{13}^{q} & m_{23}^{q} & -m_{11}-m_{22} & 0 \\
0 & 0 & 0 & 0
\end{array}\right), m_{i i} \in \mathbb{F}_{q} \forall i, m_{i j} \in \mathbb{F}_{q^{2}} \forall i \neq j\right\}
$$

whereas the image of $\ell^{\prime} \cap \mathcal{H}\left(3, q^{2}\right)$, say $\ell_{0}^{\prime}$, is contained in the space

$$
\left\{\left(\begin{array}{cccc}
0 & 0 & 0 & 0 \\
0 & 0 & 0 & 0 \\
0 & 0 & m_{33} & m_{34} \\
0 & 0 & m_{34}^{q} & -m_{33}
\end{array}\right), m_{33} \in \mathbb{F}_{q}, m_{34} \in \mathbb{F}_{q^{2}}\right\},
$$

hence their union spans the PG $(10, q)$ defined by $m_{14}=m_{24}=0$. The intersection of $\mathcal{Q}$ with this PG $(10, q)$ is the quadric with equation $m_{11}^{2}+m_{22}^{2}+m_{33}^{2}+m_{11} m_{22}+$ $m_{11} m_{33}+m_{22} m_{33}+m_{12}^{q+1}+m_{13}^{q+1}+m_{23}^{q+1}+m_{34}^{q+1}=0$, which is obviously of the same type as $\mathcal{Q}$ and of lower rank. So if $q$ is odd, then $\mathcal{O}^{\prime \alpha}$ is a partial ovoid of $\mathcal{Q}(10, q)$, if $q=2^{2 h+1}$, then the projection of $\mathcal{O}^{\prime \alpha}$ from $I$ is partial ovoid of a $\mathcal{Q}^{-}(9, q)$ and, finally, if $q=2^{2 h}$, then the projection of $\mathcal{O}^{\prime \alpha}$ from $I$ is partial ovoid of a $\mathcal{Q}^{+}(9, q)$. The stabilizer of $\mathcal{O}^{\prime}$, and hence of $\mathcal{O}^{\prime \alpha}$ is the subgroup of PGU $(4, q)$ fixing $\mathcal{H}\left(2, q^{2}\right)$ and the line $\ell$, so it is $C_{q+1} \times C_{q+1} \times \operatorname{PGL}(2, q)$, where $C_{q+1}$ denotes 
the cyclic group of order $q+1$. We want to determine whether $\mathcal{O}^{\prime \alpha}$ (or its projection) is a complete partial ovoid of the quadric in which it is embedded. Let $q$ be odd, $\perp$ be the polarity induced by $\mathcal{Q}(10, q)$ and suppose that there exists a point $P$ of $\mathcal{Q}(10, q)$, say

$$
\left(\begin{array}{cccc}
m_{11} & m_{12} & m_{13} & 0 \\
m_{12}^{q} & m_{22} & m_{23} & 0 \\
m_{13}^{q} & m_{23}^{q} & m_{33} & m_{34} \\
0 & 0 & m_{34}^{q} & -\sum_{i=1}^{3} m_{i i}
\end{array}\right)
$$

such that $P^{\perp}$ does not contain any point of $\mathcal{O}^{\prime \alpha}$. Then, we have

$$
\operatorname{Tr}\left(\begin{array}{cccc}
m_{11} & m_{12} & m_{13} & 0 \\
m_{12}^{q} & m_{22} & m_{23} & 0 \\
m_{13}^{q} & m_{23}^{q} & m_{33} & m_{34} \\
0 & 0 & m_{34}^{q} & -\sum_{i=1}^{3} m_{i i}
\end{array}\right)\left(\begin{array}{cccc}
x_{1}^{q+1} & x_{1} x_{2}^{q} & x_{1} & 0 \\
x_{1}^{q} x_{2} & x_{2}^{q+1} & x_{2} & 0 \\
x_{1}^{q} & x_{2}^{q} & 1 & 0 \\
0 & 0 & 0 & 0
\end{array}\right) \neq 0
$$

for all $x_{1}$ and $x_{2} \in \mathbb{F}_{q^{2}}$ such that $x_{1}^{q+1}+x_{2}^{q+1}+1=0$. This is equivalent to say that the two Hermitian curves, $\mathcal{H}\left(2, q^{2}\right)$, with equation $x_{1}^{q+1}+x_{2}^{q+1}+x_{3}^{q+1}=0$, and $\mathcal{H}^{\prime}$, with equation $m_{11} x_{1}^{q+1}+m_{12} x_{1}^{q} x_{2}+m_{12}^{q} x_{1} x_{2}^{q}+m_{13} x_{1}^{q} x_{3}+m_{13}^{q} x_{1} x_{3}^{q}+m_{22} x_{2}^{q+1}+$ $m_{23} x_{2}^{q} x_{3}+m_{23}^{q} x_{2} x_{3}^{q}+m_{33} x_{3}^{q+1}=0$, intersect in a set of points contained in the line $x_{3}=0$. By [18], there are only two possibilities: $\mathcal{H}^{\prime}$ is either in the pencil determined by the intersection of $\mathcal{H}\left(2, q^{2}\right)$ and the line $x_{3}=0$, or $\mathcal{H}^{\prime}$ is in the pencil determined by $\mathcal{H}\left(2, q^{2}\right)$ and the line $x_{1}+a x_{2}=0$, for every $a^{q+1}=-1$. In the first case the equation of $\mathcal{H}^{\prime}$ is of the form $k\left(x_{1}^{q+1}+x_{2}^{q+1}+x_{3}^{q+1}\right)+x_{3}^{q+1}=0$ for some $k \in \mathbb{F}_{q}$, in the latter $k\left(x_{1}^{q+1}+x_{2}^{q+1}+x_{3}^{q+1}\right)+\left(x_{1}+a x_{2}\right)^{q+1}=0$, with $k \in \mathbb{F}_{q}$. Then a point that may extend the partial ovoid must belong to one of the following two sets:

$$
\mathcal{T}_{1}=\left(\begin{array}{cccc}
k & 0 & 0 & 0 \\
0 & k & 0 & 0 \\
0 & 0 & k+1 & m_{34} \\
0 & 0 & m_{34}^{q} & -3 k-1
\end{array}\right), \quad k \in \mathbb{F}_{q}, m_{34} \in \mathbb{F}_{q^{2}},
$$

and

$$
\mathcal{T}_{2}=\left(\begin{array}{cccc}
k+1 & a^{q} & 0 & 0 \\
a & k-1 & 0 & 0 \\
0 & 0 & k & m_{34} \\
0 & 0 & m_{34}^{q} & -3 k
\end{array}\right), \quad k \in \mathbb{F}_{q}, m_{34} \in \mathbb{F}_{q^{2}}, a^{q+1}=-1 .
$$

The points of the set $\mathcal{T}_{1}$ are contained in the solid defined by the equations: $m_{14}=m_{24}=m_{12}=m_{13}=m_{23}=0, m_{11}=m_{22}$. This solid intersects $\mathcal{Q}(10, q)$ in the quadric $\mathcal{Q}^{*}$ with equation $3 m_{22}^{2}+2 m_{22} m_{33}+m_{33}^{2}+m_{34}^{q+1}=0$. The polynomial $3 m_{22}^{2}+2 m_{22} m_{33}+m_{33}^{2}$ has roots if and only if -2 is a square in $\mathbb{F}_{q}$; by the quadratic reciprocity law, it is not difficult to see that -2 is a non-square in $\mathbb{F}_{p}, p$ an odd prime, if and only if $p \equiv-1,-3 \bmod 8$. Since $\mathcal{Q}^{*}$ is the orthogonal sum of the two lines 
defined by $m_{34}=0$ and by $m_{22}=m_{33}=0$, then $\mathcal{Q}^{*}$ is hyperbolic if and only if $q=p^{2 h+1}$ and $p \equiv-1,-3 \bmod 8$, while it is elliptic for all other odd $q$. In order to extend $\mathcal{O}^{\prime \alpha}$, a point $P$ of $\mathcal{T}_{1}$ has to be non-collinear also with the points of

$$
\ell_{0}^{\prime \alpha}=\left(\begin{array}{cccc}
0 & 0 & 0 & 0 \\
0 & 0 & 0 & 0 \\
0 & 0 & 1 & b \\
0 & 0 & b^{q} & -1
\end{array}\right), \quad b^{q+1}=-1
$$

which actually lie on a conic contained in $\mathcal{Q}^{*}$. If $\mathcal{Q}^{*}$ is elliptic, we can extend the partial ovoid with the points of $\mathcal{T}_{1} \cap \mathcal{Q}^{*}$ not in that conic; if $\mathcal{Q}^{*}$ is hyperbolic, then we cannot take any point of $\mathcal{T}_{1}$.

The points of $\mathcal{T}_{2}$ that can extend the partial ovoid have to be points of the quadric, hence $6 k^{2}+m_{34}^{q+1}=0$, and they have to be non-collinear with the points of $\ell_{0}^{\prime \alpha}$, so $4 k+m_{34} b^{q}+m_{34}^{q} b \neq 0$, for all $b$ such that $b^{q+1}=-1$. If $q=3^{h}$, then a point of $\mathcal{T}_{2}$ with $m_{34}=0$ and $k \neq 0$ can extend the partial ovoid. If the characteristic of $q$ is not 3 , then we have to determine the common solutions of $6 k^{2}+m_{34}^{q+1}=0$ and $4 k+m_{34} b^{q}+m_{34}^{q} b=0$. Let $\xi:=m_{34} b^{q}$, then $\xi+\xi^{q}=-4 k$ and $\xi^{q+1}=6 k^{2}$, implying that $\xi$ is a root of $x^{2}+4 k x+6 k^{2}=0$. If -2 is a square in $\mathbb{F}_{q}$, then the roots of the equation are in $\mathbb{F}_{q}$ and we would get $2 \xi=-4 k, \xi^{2}=6 k^{2}$, hence a point of $\mathcal{T}_{2}$ with $6 k^{2}+m_{34}^{q+1}=0$ and $k \neq 0$ can extend the partial ovoid. If -2 is not a square in $\mathbb{F}_{q}$, then there exists $\xi \in \mathbb{F}_{q^{2}} \backslash \mathbb{F}_{q}$ root of $x^{2}+4 k x+6 k^{2}=0$, and so no point of $\mathcal{T}_{2}$ can extend the partial ovoid.

Suppose now $q$ to be even. If the projection of $\mathcal{O}^{\prime \alpha}$ onto a hyperplane not through $I$ is not a complete partial ovoid, then there exists a hyperplane of $\operatorname{PG}(10, q)$ through $I$ such that its intersection with $\mathcal{O}^{\prime \alpha}$ is empty. The equation of the generic hyperplane is of type $\operatorname{Tr}(A M)=0$, where $A=\left(a_{i j}\right), M=\left(m_{i j}\right)$, with $\operatorname{Tr}(M)=0, a_{14}=a_{24}=$ $m_{14}=m_{24}=0$ and, since the hyperplane contains $I, \operatorname{Tr}(A)=0$. The condition that the hyperplane does not contain any point of $\mathcal{O}^{\prime \alpha}$ implies, as before, $a_{13}=a_{23}=0$ and $A$ has to be either of the form

$$
\begin{aligned}
& \left(\begin{array}{cccc}
k & 0 & 0 & 0 \\
0 & k & 0 & 0 \\
0 & 0 & k+1 & a_{34} \\
0 & 0 & a_{34}^{q} & k+1
\end{array}\right) \quad \text { or of the form } \\
& \left(\begin{array}{cccc}
k+1 & a & 0 & 0 \\
a^{q} & k+1 & 0 & 0 \\
0 & 0 & k & a_{34} \\
0 & 0 & a_{34}^{q} & k
\end{array}\right), \quad a^{q+1}=1 .
\end{aligned}
$$

In both cases, the property of not including the points of $\ell_{0}^{\prime \alpha}$ implies that $a_{34} b^{q}+$ $a_{34}^{q} b \neq 0$ for all $b$ such that $b^{q+1}=1$. But for all $a_{34} \in \mathbb{F}_{q^{2}} \backslash\{0\}$, take $b=\lambda a_{34}$, with $\lambda=\left(\frac{1}{a_{34}^{q+1}}\right)^{\frac{1}{2}} \in \mathbb{F}_{q}$, with the obvious meaning for $x^{\frac{1}{2}}$ for $x$ in a finite field of even characteristic. So $b^{q+1}=1$ and $a_{34} b^{q}=\lambda a_{34}^{q+1} \in \mathbb{F}_{q}$ has trace 0 . Hence $\mathcal{O}^{\prime \alpha}$ is projected onto a complete partial ovoid of $\mathcal{Q}^{+}(9, q)$ if $q=2^{2 h}$ and onto a complete partial ovoid of $\mathcal{Q}^{-}(9, q)$ if $q=2^{2 h+1}$.

We summarize in the following the obtained results. 
Theorem 3 By the twisted tensor embedding of the ovoid $\mathcal{O}^{\prime}$ of $\mathcal{H}\left(3, q^{2}\right)$ obtained by derivation from the classical one, we get a partial ovoid $\Theta$ of the quadric $\mathcal{Q}$ with stabilizer $C_{q+1} \times C_{q+1} \times \operatorname{PGL}(2, q)$, where $C_{q+1}$ denotes the cyclic group of order $q+1$. When $q=2^{2 h+1}, \mathcal{Q}=\mathcal{Q}^{-}(9, q)$, while when $q=2^{2 h}, \mathcal{Q}=\mathcal{Q}^{+}(9, q)$; in both cases $\Theta$ is complete. When $q$ is odd, $\mathcal{Q}=\mathcal{Q}(10, q)$ and $\Theta$ is complete if and only if $f q=p^{2 h+1}$ and $p \equiv-1,-3 \bmod 8, p$ prime.

We now want to consider the embedding of an ovoid of $\mathcal{H}\left(3, q^{2}\right)$ that has the property to be a translation ovoid. A translation ovoid $\mathcal{O}$ of a polar space with respect to one of its points $P$ is such that there exists a collineation preserving the polar space fixing $P$, leaving invariant the generators through $P$ and acting regularly on the points of $\mathcal{O} \backslash\{P\}$. The classical ovoid is a translation ovoid with respect to any of its points; the first non-classical translation ovoid appearing in literature is the one constructed in [6]. This latter ovoid can be obtained by means of an orthogonal polarity commuting with the unitary polarity induced by $\mathcal{H}\left(3, q^{2}\right)$. The notion of commuting polarity has been originally introduced by Tits in [34], however [27] is the most exhaustive source for the unitary case. Let $\tau$ be an $\mathbb{F}_{q}$-linear collineation of order two fixing $\mathcal{H}\left(3, q^{2}\right)$ and let $\mathcal{B}$ the Baer subgeometry consisting of the points fixed by $\tau$. Let $\mathcal{Q}$ be the quadric $\mathcal{H}\left(3, q^{2}\right) \cap \mathcal{B}$, then we say that the polarity induced by $\mathcal{H}\left(3, q^{2}\right)$ and the polarity induced by $\mathcal{Q}$ are commuting. Let $\mathcal{B}$ be such that $\mathcal{Q}$ is an elliptic quadric $\mathcal{Q}^{-}(3, q)$ and let $P \in \mathcal{Q}$. The lines joining $P$ with the other points of $\mathcal{Q}$ are $(q+1)$-secant to $\mathcal{H}\left(3, q^{2}\right)$. Let $\mathcal{O}$ be the set of the points of $\mathcal{H}\left(3, q^{2}\right)$ contained in these $q^{2}$ lines. If $q$ is odd, then $\mathcal{O}$ is a translation ovoid of $\mathcal{H}\left(3, q^{2}\right)$ (see [6]). We want to embed this ovoid. From now on, assume $q$ to be odd. Let $\tau$ be the map $\left(x_{1}, x_{2}, x_{3}, x_{4}\right) \mapsto\left(x_{2}^{q}, x_{1}^{q}, x_{3}^{q}, \xi^{1-q} x_{4}^{q}\right)$, with $-1=\xi^{q+1}$. Hence $\mathcal{B}=\left\{\left(x_{1}, x_{1}^{q}, x_{3}, \xi x_{4}\right), x_{1} \in \mathbb{F}_{q^{2}}, x_{3}\right.$ and $\left.x_{4} \in \mathbb{F}_{q}\right\}$ and $\mathcal{Q}$ is the elliptic quadric of $\mathcal{B}$ defined by the equation $2 x_{1}^{q+1}+x_{3}^{2}-x_{4}^{2}=0$. Let $P=(0,0,1, \xi)$, then $\mathcal{Q} \backslash\{P\}=\left\{\left(x, x^{q}, \frac{1-2 x^{q+1}}{2},-\xi \frac{1+2 x^{q+1}}{2}\right), x \in \mathbb{F}_{q^{2}}\right\}$. By straightforward calculations, we get $\mathcal{O}=\{P\} \cup\left\{\left(x, x^{q}, \frac{1-2 x^{q+1}}{2}+\lambda,-\xi \frac{1+2 x^{q+1}}{2}+\lambda \xi\right), x \in \mathbb{F}_{q^{2}}, \lambda \in\right.$ $\left.\mathbb{F}_{q^{2}} \mid \lambda+\lambda^{q}=0\right\}$. Let $y_{1}:=\frac{1-2 x^{q+1}}{2}$ and $y_{2}:=-\frac{1+2 x^{q+1}}{2}$. Then

$$
\left.\begin{array}{rl}
P^{\alpha} & =\left(\begin{array}{cccc}
0 & 0 & 0 & 0 \\
0 & 0 & 0 & 0 \\
0 & 0 & 1 & \xi^{q} \\
0 & 0 & \xi & -1
\end{array}\right), \\
(\mathcal{O} \backslash\{P\})^{\alpha} & \\
= & \left\{\begin{array}{cccc}
x^{q+1} & x^{2} & x\left(y_{1}+\lambda^{q}\right) & x \xi^{q}\left(y_{2}+\lambda^{q}\right) \\
x^{2 q} & x^{q+1} & x^{q}\left(y_{1}+\lambda^{q}\right) & x^{q} \xi^{q}\left(y_{2}+\lambda^{q}\right) \\
x^{q}\left(y_{1}+\lambda\right) & x\left(y_{1}+\lambda\right) & y_{1}^{2}+\lambda^{q+1} & \left(y_{1}+\lambda\right) \xi^{q}\left(y_{2}+\lambda^{q}\right) \\
x^{q} \xi\left(y_{2}+\lambda\right) & x \xi\left(y_{2}+\lambda\right) & \left(y_{1}+\lambda^{q}\right) \xi\left(y_{2}+\lambda\right) & -y_{2}^{2}-\lambda^{q+1}
\end{array}\right), \\
& x \in \mathbb{F}_{q^{2}, \lambda \in \mathbb{F}_{q^{2}} \mid \lambda+\lambda^{q}=0}
\end{array}\right\} .
$$


It follows that $\mathcal{O}^{\alpha}$ is contained in the subspace of PG $(14, q)$ defined by the equations $m_{11}=m_{22}, m_{13}+\xi m_{14}=\left(m_{23}+\xi m_{24}\right)^{q}$, hence it is contained in a PG $(11, q)$. This space is the polar of the plane

$$
\pi:=\left\{\left(\begin{array}{cccc}
m_{11} & 0 & m_{13} & \xi^{q} m_{13} \\
0 & -m_{11} & -m_{13}^{q} & -\xi^{q} m_{13}^{q} \\
m_{13}^{q} & -m_{13} & 0 & 0 \\
\xi m_{13}^{q} & -\xi m_{13} & 0 & 0
\end{array}\right), m_{11} \in \mathbb{F}_{q}, m_{13} \in \mathbb{F}_{q^{2}}\right\}
$$

that is a plane that intersects $\mathcal{Q}(14, q)$ in the line

$$
\ell:=\left\{\left(\begin{array}{cccc}
0 & 0 & m_{13} & \xi^{q} m_{13} \\
0 & 0 & -m_{13}^{q} & -\xi^{q} m_{13}^{q} \\
m_{13}^{q} & -m_{13} & 0 & 0 \\
\xi m_{13}^{q} & -\xi m_{13} & 0 & 0
\end{array}\right), m_{13} \in \mathbb{F}_{q^{2}}\right\}
$$

Hence $\pi^{\perp} \cap \mathcal{Q}(14, q)$ is a cone with vertex $\ell$ and base either a $\mathcal{Q}^{+}(9, q)$ or $\mathcal{Q}^{-}(9, q)$. The space $\ell^{\perp}$ has equation $m_{13}+\xi m_{14}=\left(m_{23}+\xi m_{24}\right)^{q}$ and it is a cone with vertex $\ell$ and base a $\mathcal{Q}(10, q)$ contained in the space

$$
\left.\begin{array}{c}
\left\{\left(\begin{array}{cccc}
m_{11} & m_{12} & -\xi m_{14}+\xi^{q} m_{24}^{q} & m_{14} \\
m_{12}^{q} & m_{22} & 0 & m_{24} \\
-\xi^{q} m_{14}^{q}+\xi m_{24} & 0 & m_{33} & m_{34} \\
m_{14}^{q} & m_{24}^{q} & m_{34}^{q} & -m_{11}-m_{22}-m_{33}
\end{array}\right),\right. \\
m_{i i} \in \mathbb{F}_{q} \forall i, m_{i j} \in \mathbb{F}_{q^{2}}, \forall i \neq j
\end{array}\right\} .
$$

Let

$$
R=\left(\begin{array}{cccc}
1 & 0 & 0 & 0 \\
0 & -1 & 0 & 0 \\
0 & 0 & 0 & 0 \\
0 & 0 & 0 & 0
\end{array}\right)
$$

then $\pi=\langle R, \ell\rangle$. The space $R^{\perp}$ has equation $m_{11}=m_{22}$ and it intersects $\mathcal{Q}(10, q)$ in the space

$$
\begin{gathered}
\left\{\left(\begin{array}{cccc}
m_{11} & m_{12} & -\xi m_{14}+\xi^{q} m_{24}^{q} & m_{14} \\
m_{12}^{q} & m_{11} & 0 & m_{24} \\
-\xi^{q} m_{14}^{q}+\xi m_{24} & 0 & m_{33} & m_{34} \\
m_{14}^{q} & m_{24}^{q} & m_{34}^{q} & -2 m_{11}-m_{33}
\end{array}\right),\right. \\
\left.m_{i i} \in \mathbb{F}_{q} \forall i, m_{i j} \in \mathbb{F}_{q^{2}} \forall i \neq j\right\} .
\end{gathered}
$$


Hence we see that $\mathcal{Q}(10, q) \cap R^{\perp}$ is the quadric with equation $3 m_{11}^{2}+m_{33}^{2}+$ $2 m_{11} m_{33}+m_{12}^{q+1}+m_{34}^{q+1}-\operatorname{Tr}\left(\xi^{2} m_{14} m_{24}\right)=0$.

The polynomial $3 m_{22}^{2}+m_{33}^{2}+2 m_{22} m_{33}$ is irreducible if and only if -2 is a nonsquare in $\mathbb{F}_{p}$ and $q=p^{2 h+1}$. As observed before, -2 is a non-square in $\mathbb{F}_{p}$ if and only if $p \equiv-1,-3 \bmod 8$. Hence the line defined by the equations $m_{i j}=0, \forall i \neq j$, is non-isotropic if and only if $p \equiv-1,-3 \bmod 8$ and $q=p^{2 h+1}$, and it is secant for all other odd prime powers $q$. The subspace defined by $m_{i i}=0, i=1,3$, intersects $\mathcal{Q}(10, q) \cap R^{\perp}$ in a $\mathcal{Q}^{+}(7, q)$, since we get the orthogonal sum of two non-isotropic lines and a $\mathcal{Q}^{+}(3, q)$. Hence, the quadric $P^{\perp} \cap \mathcal{Q}(10, q)$ is a $\mathcal{Q}^{-}(9, q)$ if $p \equiv-1,-3$ $\bmod 8$ and $q=p^{2 h+1}$, and a $\mathcal{Q}^{+}(9, q)$ for all other odd prime powers $q$.

Thus, projecting $\mathcal{O}^{\alpha}$ from $\ell$, we obtain a partial ovoid of size $q^{3}+1$ of $\mathcal{Q}^{-}(9, q)$ for $p \equiv-1,-3 \bmod 8$ and $q=p^{2 h+1}$ or of $\mathcal{Q}^{+}(9, q)$ for all the other odd prime powers $q$. Suppose that this partial ovoid is not complete, hence there should be a point $R$ such that $R^{\perp} \cap \mathcal{O}^{\alpha}=\emptyset$. This implies that there is a Hermitian surface having empty intersection with $\mathcal{O}$. It is more convenient for our calculations to consider the ovoid as it appears in [6], namely $\mathcal{O}^{\prime}:=\left\{\left(1, t, t^{q}, t^{q+1}+\lambda\right), t \in\right.$ $\left.\mathbb{F}_{q^{2}}, \lambda \in \mathbb{F}_{q^{2}} \mid \lambda+\lambda^{q}=0\right\} \cup\{(0,0,0,1)\}$. For odd $q, \mathcal{O}^{\prime}$ is a translation ovoid of the Hermitian surface with equation $x_{1} x_{4}^{q}+x_{1}^{q} x_{4}-x_{2}^{q+1}-x_{3}^{q+1}=0$ and it is projectively equivalent to $\mathcal{O}$. Let $\operatorname{Tr}$ be the trace map from $\mathbb{F}_{q^{2}}$ to $\mathbb{F}_{q}$ and observe that $\mathcal{O}^{\prime}=\left\{\left(1, t, t^{q}, u\right), t \in \mathbb{F}_{q^{2}}, u \in \mathbb{F}_{q^{2}} \mid \operatorname{Tr}(u)=\operatorname{Tr}\left(t^{q+1}\right)\right\} \cup\{(0,0,0,1)\}$. Let $\mathcal{H}$ be a (possibly singular) Hermitian surface of PG $\left(3, q^{2}\right)$ such that $\mathcal{H} \cap \mathcal{O}^{\prime}=\emptyset$. Let $A=\left(a_{i j}\right)$ be the Hermitian matrix defining $\mathcal{H}$, then $\mathcal{H} \cap\left(\mathcal{O}^{\prime} \backslash\{(0,0,0,1)\}\right)$ is given by the solutions of the system:

$$
\left\{\begin{array}{l}
a_{11}+\operatorname{Tr}\left(a_{12} t^{q}+a_{13} t+a_{14} u^{q}\right)+a_{22} t^{q+1}+\operatorname{Tr}\left(a_{23} t^{2}+a_{24} t u^{q}\right)+a_{33} t^{q+1} \\
\quad+\operatorname{Tr}\left(a_{34} t^{q} u^{q}\right)+a_{44} u^{q+1}=0, \\
\operatorname{Tr}(u)=\operatorname{Tr}\left(t^{q+1}\right) .
\end{array}\right.
$$

On $\mathbb{F}_{q}$, this is a system in four variables with two quadratic non-homogeneous equations. If we consider the homogenized equations, we get two quadratic equations in five variables, hence, by the Chevalley-Warning Theorem, that system has always at least one solution in $\mathrm{PG}(4, q)$. In order to get no solutions in $\operatorname{AG}(4, q)$, we should get at least one solution in the hyperplane at infinity, hence we must find at least one (projective) solution for:

$$
\left\{\begin{array}{l}
a_{22} t^{q+1}+\operatorname{Tr}\left(a_{23} t^{2}+a_{24} t u^{q}\right)+a_{33} t^{q+1}+\operatorname{Tr}\left(a_{34} t^{q} u^{q}\right)+a_{44} u^{q+1}=0, \\
0=\operatorname{Tr}\left(t^{q+1}\right) \Longleftrightarrow t=0
\end{array}\right.
$$

hence $a_{44}=0$. This implies that $\mathcal{H}$ contains the point $(0,0,0,1)$ and so a Hermitian surface with empty intersection with $\mathcal{O}^{\prime}$ does not exist. It follows that the partial ovoid obtained by the projection of $\mathcal{O}^{\alpha}$ is complete. The group fixing $\mathcal{O}$ is isomorphic to the one fixing the complete partial ovoid and it contains an elementary abelian group of order $q^{3}$ fixing $P$ and acting transitively on the other points (see [6]).

So, we have proved the following.

Theorem 4 Let $\mathcal{O}$ be the translation ovoid of $\mathcal{H}\left(3, q^{2}\right), q$ odd, obtained by a commuting orthogonal polarity of $\mathrm{PG}(3, q)$. By the twisted tensor embedding of $\mathcal{O}$, 
we get a complete partial ovoid $\Theta$ of the quadric $\mathcal{Q}$, where $\mathcal{Q}=\mathcal{Q}^{-}(9, q)$ for $q=p^{2 h+1}, p$ a prime and $p \equiv-1,-3 \bmod 8$, while $\mathcal{Q}=\mathcal{Q}^{+}(9, q)$ for all the other odd prime powers $q$. The stabilizer of $\Theta$ contains an elementary abelian group of order $q^{3}$ fixing a point of $\Theta$ and acting transitively on the other points.

\section{References}

1. Blokhuis, A., Moorhouse, G.E.: Some p-ranks related to orthogonal spaces. J. Algebr. Comb. 4, 295316 (1995)

2. Conway, J.H., Kleidman, P.B., Wilson, R.A.: New families of ovoids in $O_{8}^{+}$. Geom. Dedic. 26, 157170 (1988)

3. Cooperstein, B.N., Thas, J.A., Van Maldeghem, H.: Hermitian Veroneseans over finite fields. Forum Math. 16, 365-381 (2004)

4. Cossidente, A.: On twisted tensor product group embeddings and the spin representation of symplectic groups: the case $q$ odd. ISRN Geom. 2011, 694605 (2011), 6 pp.

5. Cossidente, A., King, O.H.: Twisted tensor product group embeddings and complete partial ovoids on quadrics in PG(2t $-1, q)$. J. Algebra 273, 854-868 (2004)

6. Cossidente, A., Penttila, T.: Hemisystems on the Hermitian surface. J. Lond. Math. Soc. 72, 731-741 (2005)

7. Cossidente, A., Siciliano, A.: On the geometry of the Hermitian matrices of order three over finite fields. Eur. J. Comb. 22, 1047-1051 (2001)

8. De Beule, J., Klein, A., Metsch, K., Storme, L.: Partial ovoids and partial spreads in symplectic and orthogonal polar spaces. Eur. J. Comb. 29, 261-272 (2008)

9. Dembowski, P.: Finite Geometries. Springer, Berlin (1968)

10. Dye, R.: Partitions and their stabilizers for line complexes and quadrics. Ann. Math. 114, 173-194 (1977)

11. Gunawardena, A., Moorhouse, G.E.: The non-existence of ovoids in $O_{9}(q)$. Eur. J. Comb. 18, 171173 (1997)

12. Harris, J.: Algebraic Geometry: A First Course. GTM, vol. 133. Springer, Berlin (1992)

13. Hasset, B.: Introduction to Algebraic Geometry. Cambridge University Press, Cambridge (2007)

14. Hirschfeld, J.W.P.: Finite Projective Spaces of Three Dimension. Oxford University Press, London (1986)

15. Hirschfeld, J.W.P., Thas, J.A.: General Galois Geometries. Oxford University Press, Oxford (1991)

16. Kantor, W.M.: Ovoids and translation planes. Can. J. Math. 36, 1195-1207 (1982)

17. Kantor, W.M.: Spreads translation planes and Kerdock sets I. SIAM J. Algebr. Discrete Methods 3, 151-165 (1982)

18. Kestenband, B.C.: Unital intersections in finite projective planes. Geom. Dedic. 11, 107-117 (1981)

19. Lunardon, G.: Planar fibrations and algebraic subvarieties of the Grassmann variety. Geom. Dedic. 16, 291-313 (1984)

20. Lunardon, G.: Segre varieties and ovoids of the polar space $Q^{+}(7, q)$. Geom. Dedic. 20, 121-131 (1986)

21. Lunardon, G.: Normal spreads. Geom. Dedic. 75, 245-261 (1999)

22. Moorhouse, G.E.: Ovoids from the $E_{8}$ root lattice. Geom. Dedic. 46, 287-297 (1993)

23. Muir, T.: A Treatise on the Theory of Determinants. Dover, New York (1960)

24. Payne, S.E., Thas, J.A.: Finite Generalized Quadrangles. Pitman, London (1984)

25. Pepe, V.: On the algebraic variety $\mathcal{V}_{r, t}$. Finite Fields Appl. 17, 343-349 (2011)

26. Segre, B.: Teoria di Galois, fibrazioni proiettive e geometrie non desarguesiane. Ann. Mat. Pura Appl. 64, 1-76 (1964)

27. Segre, B.: Forme e geometrie Hermitiane con particolare riguardo al caso finito. Ann. Mat. Pura Appl. 70, 1-201 (1965)

28. Steinberg, R.: Representations of algebraic groups. Nagoya Math. J. 22, 33-56 (1963)

29. Taylor, D.E.: The Geometry of the Classical Groups. Heldermann, Berlin (1992)

30. Thas, J.A.: Ovoidal translation planes. Arch. Math. 23, 110-112 (1971)

31. Thas, J.A.: Polar spaces, generalized hexagons and perfect codes. J. Comb. Theory A 29, 87-93 (1980)

32. Thas, J.A.: Ovoids and spreads of finite classical polar spaces. Geom. Dedic. 10, 135-144 (1981) 
33. Thas, J.A., Payne, S.E.: Spreads and ovoids in finite generalized quadrangle. Geom. Dedic. 52, 227253 (1994)

34. Tits, J.: Sur certaines classes d'espaces homogènes de groupes de Lie. Acad. R. Belg. Cl. Sci. Mém. Coll. in $8^{\circ}$ 29(3), 268 (1955)

35. Tits, J.: Sur la trialité et certains groupes qui a'en déduisent. Inst. Hautes Études Sci. Publ. Math. 2, 13-60 (1959)

36. Tits, J.: Les groupes simples de Suzuki et de Ree. In: Séminaire Bourbaki, Exp. No. 210, vol. 6, pp. 65-82. Soc. Math. France, Paris (1995) 